\title{
What can spike train distances tell us about the neural code?
}

\author{
Daniel Chicharro $^{\mathrm{a}, \mathrm{b}, 1, *}$, Thomas Kreuz ${ }^{\mathrm{b}, \mathrm{c}}$, Ralph G. Andrzejak ${ }^{\mathrm{a}}$ \\ ${ }^{a}$ Dept. of Information and Communication Technologies, Universitat Pompeu Fabra, Barcelona, \\ Spain. \\ ${ }^{b}$ Institute for Nonlinear Science, University of California, San Diego, La Jolla, CA, USA. \\ ${ }^{c}$ Institute for Complex Systems, CNR, Sesto Fiorentino, Italy.
}

\begin{abstract}
Time scale parametric spike train distances like the Victor and the van Rossum distances are often applied to study the neural code based on neural stimuli discrimination. Different neural coding hypotheses, such as rate or coincidence coding, can be assessed by combining a time scale parametric spike train distance with a classifier in order to obtain the optimal discrimination performance. The time scale for which the responses to different stimuli are distinguished best is assumed to be the discriminative precision of the neural code. The relevance of temporal coding is evaluated by comparing the optimal discrimination performance with the one achieved when assuming a rate code.

We here characterize the measures quantifying the discrimination performance, the discriminative precision, and the relevance of temporal coding. Furthermore, we evaluate the information these quantities provide about the neural code. We show that the discriminative precision is too unspecific to be interpreted in terms of the time scales relevant for encoding. Accordingly, the time scale parametric nature of the distances is mainly an advantage because it allows maximizing the discrimination performance across a whole set of measures with different sensitivities determined by the time scale parameter, but not due to the possibility to examine the temporal properties of the neural code.
\end{abstract}

Keywords: spike trains, spike train distances, discrimination, mutual information, precision, temporal coding, neural coding

${ }^{*}$ Corresponding Author:

Email address: daniel.chicharro@upf .edu (Daniel Chicharro )

URL: http: //www. cns.upf.edu/daniel/ (Daniel Chicharro )

${ }^{1}$ Adress: Roc Boronat 138, 08018 Barcelona, Spain, Phone: 0034 93-542-1371 


\section{Introduction}

In the study of the responses of sensory neurons to external stimuli, in analogy to communication channels (Shannon, 1948; Cover and Thomas, 2006), the mutual information has been used to examine the capacity of neurons to convey information about the stimulus (see Rieke et al., 1997, and references therein). Alternatively, one can use a decoding strategy to see how well the stimuli can be discriminated from the elicited spike trains (see Quian Quiroga and Panzeri, 2009, for a comparison of information theory and decoding approaches). We here study a discrimination analysis that combines a measure of dissimilarity of the spike trains with a classifier to assign each spike train to the stimulus which is most likely to have elicited it. This allows one to calculate a measure of discrimination performance, like the mutual information between the stimuli and the predicted stimuli.

A wide variety of methods have been applied to quantify the similarity between spike trains (e. g. Mainen and Sejnowski, 1995; Victor and Purpura, 1996; Hunter et al., 1998; van Rossum, 2001; Schreiber et al., 2003; Kreuz et al., 2007). A particular class of measures designed to study the neural code are time scale parametric spike train distances, mainly represented by the distances proposed by Victor and Purpura (1996) and van Rossum (2001). These spike train distances have in common that they depend on some parameter $\tau$ that determines the temporal scales in the spike trains to which the distance is sensitive. For the limit $\tau \rightarrow \infty$ these distances are only sensitive to the number of spikes in each spike train, while in the limit $\tau \rightarrow 0$ they are spike coincidence detectors. These limits reflect the assumption of a rate code and a coincidence code, respectively. When used for the discrimination analysis, the time scale parametric spike train distances provide a quantification of the discriminative precision, associated with the optimal time scale $\tau^{*}$ for which the maximal mutual information between the stimuli and the predicted stimuli is found. This precision is conceptually different from the single spike precision, that indicates the jitter of each reliable spike from trial to trial.

Discrimination analysis has been applied to study experimental data using different dissimilarity measures. For example, the Victor distance (see Victor, 2005, for a review) has been applied to study the precision in the visual system (e. g. Victor and Purpura, 1998; Mechler et al., 1998), the auditory system (e. g. Machens et al., 2001) or the olfactory system (e. g. Macleod et al., 1998). The van Rossum distance has recently been used in a series of studies on the time scale of discrimination of natural sounds in songbirds (e. g. Narayan et al., 2006; Wang et al., 2007). The correlation-based reliability measure of Schreiber et al. (2003) has also been used to analyze auditory responses (Wang et al., 2007). A common conclusion of many of these studies is that the optimal time scale found indicates that the temporal structure of the responses and not only the total spike counts are relevant 
for the neural encoding of the stimuli.

Apart from applications to experimental data, the time scale parametric spike train distances have been previously characterized (Victor and Purpura, 1997; Schrauwen and van Campenhout, 2007; Kreuz et al., 2007; Dauwels et al., 2009; Kreuz et al., 2009; Paiva et al., 2010; Kreuz et al., 2011). Victor and Purpura (1997) showed examples of simulated data for which the discrimination analysis resulted in an optimal time scale consistent with a priori expectations given the construction of the spike trains. The main focus of other studies was to compare the sensitivity of the time scale parametric distances and other measures of spike train similarity to discriminate between different stimuli, without addressing the meaning of the optimal time scale at which the sensitivity was optimized (e. g. Kreuz et al., 2007; Paiva et al., 2010). We here follow Victor and Purpura (1997) and consider the time scale parametric spike train distances integrated in the discrimination analysis. We are interested in examining how informative the mutual information and discriminative precision are about the neural code. In particular, we question what can be concluded from the discriminative precision about the time scales in which information is contained in the responses.

The remainder of the article is organized as follows: We first describe the discrimination analysis used to calculate the mutual information and discriminative precision (Section 2.1), as well as the measures of spike train dissimilarity studied (Section 2.2). We apply the measures to simulated time-independent Poisson processes (Section 3.1), and the discrimination analysis to time-dependent Poisson processes (Section 3.2). Examples of time-dependent Poisson processes are presented to study the dependence of the mutual information and the optimal time scale on different factors. We examine the dependence on the measure and classifier used for the discrimination analysis. We simulate different examples representing possible types of responses to the transient presentation of a constant stimuli and study the dependence of the mutual information and the optimal time scale on the length of the after-transient interval included in the discrimination analysis.

Furthermore, apart from the simulated data, in Section 3.3 we also use exemplary experimental recordings. These data were delivered via http://neurodatabase.org, a neuroinformatics resource funded by the Human Brain Project (Gardner, 2004). They consist in spike trains elicited by transient constant stimuli. We examine the dependence on the measure and classifier in Section 3.3.1, and the temporal accumulation of information obtained from increasing the length of the recordings used to calculate the spike train dissimilarity measures (Section 3.3.2). We also analyze the temporal distribution of information and the redundancy between the information contained in different intervals of the recordings (Section 3.3.3), and examine how this temporal distribution is reflected in the optimal time scale. We consider the degree to which the complex temporal structure of the spike trains is 
well represented in a single quantity, namely the optimal time scale $\tau^{*}$ associated with the discriminative precision. Finally, in Section 4 we discuss our results and how to interpret the quantities obtained from the discrimination analysis.

\section{Methods}

\subsection{Discrimination analysis}

The mutual information $I(\mathbf{R}, S)$ (Cover and Thomas, 2006) is used to characterize the encoding of the stimuli $S$ by the responses $\mathbf{R}$ elicited by them. For the spike trains, the space of $\mathbf{R}$ is determined by the times of the spikes. If the spike trains are binned in $N_{b}$ bins and $r_{i}$ is the number of spikes in each bin, $\mathbf{R}=\left\{r_{1}, r_{2}, \ldots r_{N_{b}}\right\}$ will have a high dimensionality for small bins. The mutual information is calculated as:

$$
I(\mathbf{R}, S)=\sum_{\mathbf{R}} \sum_{S} p(\mathbf{R}, S) \log \frac{p(\mathbf{R}, S)}{p(\mathbf{R}) p(S)},
$$

where $p(\mathbf{R}, S)$ and $p(\mathbf{R}), p(S)$ are the joint and marginal distributions, respectively. The reliable estimation of this quantity from experimental data is hindered by the curse of dimensionality caused by sampling limitations (see for example Borst and Theunissen, 1999; Paninski, 2003; Victor, 2006; Panzeri et al., 2007, for a discussion of different estimation techniques and bias corrections).

One of the alternatives to estimate $I(\mathbf{R}, S)$ consists in using a classifier to predict to which stimulus a response pertains. Given $N$ distinct stimuli $\{S\}=$ $S_{1}, \ldots, S_{i}, \ldots, S_{N}$ and $M$ trials for each stimulus, a decoding algorithm assigns each spike train to one of the stimuli $\{S\}$ resulting in a confusion matrix $\mathcal{C}\left(S_{k}^{P}, S_{l}\right)$ that indicates the number of times a spike train corresponding to a trial of the stimulus $S_{l}$ is assigned to the stimulus $S_{k}^{P}$. The confusion matrix is normalized to a probability matrix $P\left(S_{k}^{P}, S_{l}\right)$ dividing by the total number of spike trains $N M$. The decoding performance can be quantified by the mutual information $I\left(S^{P}, S\right)$ between the stimuli and the predicted stimuli (Quian Quiroga and Panzeri, 2009), analogously to Equation 1. Since the classification is based only on $\mathbf{R}, S^{P}$ is conditionally independent from $S$ given $\mathbf{R}$, that is $S \rightarrow \mathbf{R} \rightarrow S^{P}$ forms a Markov Chain. In consequence, the data processing inequality (Cover and Thomas, 2006) assures that $I\left(S^{P}, S\right) \leq I(\mathbf{R}, S)$. Therefore, the mutual information obtained from the classifier is a lower bound for the total amount of information between the stimuli and the responses.

Different types of classifiers have been proposed depending on the nature and assumptions made about the stimuli and responses (e. g. Bialek et al., 1991; Rolls et al., 1997). The discrimination analysis proposed by Victor and Purpura (1996, 
1997) is based on a classifier in which the spike train distances are used to compute the distance from a spike train $s_{l, j^{\prime}}$ to a predicted stimulus $S_{k}^{P}$ as

$$
d\left(S_{k}^{P}, s_{l, j^{\prime}} ; \tau\right)=\left[\left\langle\left(D\left(s_{k, j}, s_{l, j^{\prime}}\right)[\tau]\right)^{z}\right\rangle_{j}\right]^{1 / z},
$$

so that the average of the spike train distances $D\left(s_{k, j}, s_{l, j^{\prime}}\right)[\tau]$ between the spike train $s_{l, j^{\prime}}$, corresponding to the $j^{\prime}$-th trial of the $l$-th stimulus, and all the trials $j=$ $1, \ldots M$ of the $k$-th stimulus is calculated (note that trial $j=j^{\prime}$ is excluded if $k=l$ ). The classification is carried out by assigning each spike train to the stimulus that minimizes this average distance. The classification depends on the parameter $\tau$, leading to a whole range of, in general, different confusion matrices. The parameter $z$ determines which geometry is considered for the average. For positive exponents the preeminent contribution comes from the larger distances, while for negative exponents the closest spike trains have a higher weight. The differences in the confusion matrices obtained for different exponents can help to infer the structure of the clusters of trials of the same stimulus and consequently the variability of the spike trains (Victor and Purpura, 1997). The mutual information between the actual and the assigned stimuli, given the probability matrix $P\left(S_{k}^{P}, S_{l} ; \tau\right)$, is obtained for each time scale:

$$
I\left(S^{P}, S ; \tau\right)=\sum_{k=1}^{N} \sum_{l=1}^{N} P\left(S_{k}^{P}, S_{l} ; \tau\right) \log \frac{P\left(S_{k}^{P}, S_{l} ; \tau\right)}{P\left(S_{k}^{P} ; \tau\right) P\left(S_{l} ; \tau\right)} .
$$

The time scale $\tau^{*}$ for which the maximal mutual information $I_{\max }\left(S^{P}, S\right)$ is found gives the best discrimination of the stimuli. The percentage reduction of uncertainty is given by:

$$
I^{*}\left(S^{P}, S ; \tau\right)=\frac{I\left(S^{P}, S ; \tau\right)}{H(S)},
$$

where $H(S)$ is the entropy of the stimuli distribution (Cover and Thomas, 2006)

$$
H(S)=-\sum_{l=1}^{N} P\left(S_{l}\right) \log P\left(S_{l}\right) .
$$

The measure $I^{*}\left(S^{P}, S ; \tau\right)$ is normalized in the range [0 1], which facilitates the comparison among results obtained for different confusion matrices, and furthermore it better quantifies the goodness of the decoding (Borst and Theunissen, 1999). Since the stimuli distribution $P(S)$ is well known, $H(S)$ can be calculated exactly, so that $I^{*}\left(S^{P}, S ; \tau\right)$ is also a lower bound of $I^{*}(\mathbf{R}, S)$, defined analogously to Equation 4. 
The estimation of the mutual information with the classifier avoids the bias caused by the estimation of probability distributions in high-dimensional spaces. However, one has to correct for the upward bias associated with the baseline level of correct classifications that would already result from a random assignment of the trials to a predicted stimulus. This bias is estimated by a random resampling of the spike trains. The trials are shuffled across stimuli and the average mutual information is calculated across multiple repetitions of the random shuffling. This correction is subtracted from the mutual information calculated for the original set, and the mutual information is considered different from zero only for positive values. We provide an estimate of the variability of $I_{\max }\left(S^{P}, S\right)$ with its standard deviation across independent realizations of the discrimination analysis in the case of simulated data and bootstrapping realizations for experimental data. For the latter the standard deviation depends on the percentage of trials left out for each realization and the lack of independence of different realizations does not allow to directly estimate the standard error of the mean.

While $I_{\text {max }}^{*}\left(S^{P}, S\right)$ is simply the maximum of $I^{*}\left(S^{P}, S ; \tau\right)$, getting the optimal time scale $\tau^{*}$ is more complicated. This is because $I^{*}\left(S^{P}, S ; \tau\right)$ can have piecewise constant intervals (e. g. Victor and Purpura, 1996; Di Lorenzo and Victor, 2003; Roussin et al., 2008; Huetz et al., 2006, 2009). Different criteria have been chosen in experimental studies that apply the discrimination analysis, but often there is not an explicit explanation of how to calculate $\tau^{*}$. In Huetz et al. (2006) and Wohlgemuth and Ronacher (2007), the average of $\tau$ is taken when a plateau is obtained. In Roussin et al. (2008) they comment that the influence of temporal coding may be underestimated because they consider as rate coding any case in which $I_{\text {max }}^{*}\left(S^{P}, S\right)$ is obtained for $\tau=\infty$, even if lower time scales provide the same information. Instead of using the position of the peak to indicate the discriminative precision, Reich et al. (2001b) use $\tau_{c u t}$, the minimal time scale at which half of the maximal information is retrieved.

We here follow two different criteria, each emphasizing different aspects of the shape of $I\left(S^{P}, S ; \tau\right)$ and its estimation. For simulated data, we can generate $K$ independent realizations of the set of trials for each stimuli, and consider the average and the variability of the results of the discrimination analysis across realizations. For each realization we retrieve a value of $\tau^{*}$ as the mean of all $\tau$ values for which $I_{\max }^{*}\left(S^{P}, S\right)$ is obtained. Accordingly, we report the average of $\left\langle\tau^{*}\right\rangle$ across realizations and we quantify its variability by the range of $\tau^{*}$. Alternatively, we calculate the average of $I^{*}\left(S^{P}, S ; \tau\right)$ for the different realizations, and then we calculate $\tau_{\langle I\rangle}^{*}$ only once from the average shape. In this case we additionally report the interval containing values of $I^{*}\left(S^{P}, S ; \tau\right)$ higher than a given percentage of the maximum (Wohlgemuth and Ronacher, 2007). Since the maximum and mean 
operations are not commutative, $\left\langle\tau^{*}\right\rangle$ and $\tau_{\langle I\rangle}^{*}$ will generally not coincide. Furthermore, while for the first calculation the range of $\tau^{*}$ quantifies the variability of the estimator, in the second case the interval of high values is related to the flatness of $I^{*}\left(S^{P}, S ; \tau\right)$. The same procedure can be applied for experimental data carrying out the calculations across $K$ bootstrapping realizations instead of independently generated data. For each bootstrapping realization the discrimination analysis is repeated randomly selecting a subset $M^{\prime}$ of the trials for each stimulus.

Apart from $\tau^{*}$, to analyze the contribution of temporal coding it is necessary to compare the maximum mutual information $I_{\text {max }}^{*}\left(S^{P}, S\right)$ with the information that can be retrieved based only on a rate code, $I_{\text {count }}^{*}\left(S^{P}, S\right)$. One can calculate an index of temporal coding like the percentage of increase (e. g. Mechler et al., 1998; Di Lorenzo and Victor, 2003)

$$
\Theta=\frac{I_{\text {max }}^{*}\left(S^{P}, S\right)-I_{\text {count }}^{*}\left(S^{P}, S\right)}{I_{\text {count }}^{*}\left(S^{P}, S\right)} .
$$

\subsection{Spike train distances}

We here describe two spike train distances, $D_{\mathrm{V}}$ proposed by Victor and Purpura (1996), $D_{\mathrm{R}}$ by van Rossum (2001), and a dissimilarity measure $D_{\mathrm{S}}$ based on the correlation-based spike train similarity measure proposed by Schreiber et al. (2003). We furthermore describe the calculation of a spike train distance $D_{\mathrm{B}}$ based on binning (Schnupp et al., 2006). These measures quantify the degree of dissimilarity of two spike trains, with spikes occurring at times $\left\{t_{i}^{x, y}\right\}=t_{1}^{x, y}, t_{2}^{x, y}, \ldots, t_{n_{x, y}}^{x, y}$, where $n_{x, y}$ denote the total number of spikes in each train.

\subsubsection{Victor distance $D_{V}$}

Victor and Purpura (1996) introduced different families of distances each motivated by different neurobiological mechanisms. These distances are all based on the point process nature of spike trains. We use only the distance denoted $D^{\text {spike }}[q]$, which we denote as $D_{\mathrm{V}}\left[\tau_{\mathrm{V}}\right]$. Among the distances proposed by Victor and Purpura (1996), this one is the most similar to the other measures considered here. The motivation for $D_{\mathrm{V}}$ was to capture the behavior of neurons as coincidence detectors. It is defined as the minimal total cost of a sequence of elementary steps that transform one spike train into another. The allowed operations are insertion, deletion, and temporal shift of a spike. While the cost of deletion and insertion is fixed to 1 , the cost of a shift by $\Delta t$ is $q|\Delta t|$, where $q$ is the cost per time unit. For a shift of length $|\Delta t|=\frac{2}{q}$ the cost of shifting is equal to the one of deleting the pair of spikes. Therefore, $\frac{2}{q}$ is the maximal separation of the spikes for which the shift operation is preferred. Given that, we here use a parameter in units of time defined as $\tau_{\mathrm{V}}=\frac{2}{q}$. 
Table 1: Limits of the spike train dissimilarity measures

\begin{tabular}{ccc}
\hline \hline Measure & $\tau=\infty$ & $\tau=0$ \\
\hline$D_{\mathrm{V}}$ & $\left|n_{x}-n_{y}\right|$ & $n_{x}+n_{y}$ \\
$D_{\mathrm{V}}^{*}$ & $\frac{\left|n_{x}-n_{y}\right|}{n_{x}+n_{y}}$ & 1 \\
$D_{\mathrm{R}}$ & $\left(n_{x}-n_{y}\right)^{2}$ & $n_{x}+n_{y}$ \\
$D_{\mathrm{S}}$ & 0 & 1 \\
$D_{\mathrm{B}}$ & $\left(n_{x}-n_{y}\right)^{2}$ & $n_{x}+n_{y}$
\end{tabular}

Each value of $\tau_{\mathrm{V}}$ identifies one member of the family of distances. At one extreme, $D_{\mathrm{V}}\left[\tau_{\mathrm{V}}=\infty\right]=\left|n_{x}-n_{y}\right|$ is only sensitive to the difference in the total number of spikes in each train. Accordingly, it quantifies the similarity of the spike trains assuming a rate code. The distance $D_{\mathrm{V}}$ monotonically increases for $\tau_{\mathrm{V}}$ decreasing. In the other extreme, assuming that the sampling resolution is high enough so that there are no coincident spikes, $D_{\mathrm{V}}\left[\tau_{\mathrm{V}}=0\right]=n_{x}+n_{y}$. This magnitude of the distances for $\tau_{\mathrm{V}} \rightarrow 0$ is not normalized, but depends on the total number of spikes $n=n_{x}+n_{y}$. A normalized version of the Victor distance, $D_{\mathrm{V}}^{*}=\frac{D_{V}}{n_{x}+n_{y}}$, was introduced by Kreiman et al. (2000), resulting in the limits indicated in Table 1.

\subsection{2. van Rossum distance $D_{R}$}

The second spike train distance studied here was introduced by van Rossum (2001). This distance operates on continuous signals obtained by convolution of the spike trains with a truncated exponential function:

$$
f_{x, y}(t)=\sum_{i}^{n_{x, y}} u\left(t-t_{i}^{x, y}\right) e^{-\left(t-t_{i}^{x, y}\right) / \tau_{\mathrm{R}}},
$$

where $u$ is the Heaviside step function $\left(u\left(t-t_{i}\right)=0\right.$ if $t<t_{i}$ and $u\left(t-t_{i}\right)=1$ if $\left.t \geq t_{i}\right)$. Similarly to $\tau_{\mathrm{V}}$, the time constant $\tau_{\mathrm{R}}$ determines the precision to which the distance is sensitive to. The truncated exponential convolution kernel is motivated by its causality and its correspondence to the shape of postsynaptic currents. Given two continuous signals the distance is calculated as the $L^{2}$ norm

$$
D_{R}\left[\tau_{\mathrm{R}}\right]=\frac{1}{\tau_{\mathrm{R}}} \int_{0}^{\infty}\left[f_{x}-f_{y}\right]^{2} d t .
$$

As the Victor distance, the van Rossum distance avoids a coarse binning procedure. The time constant of the exponential function $\tau_{\mathrm{R}}$ determines the interval for which relevant contributions to the integral last. Note that this interval generally extends 
also beyond the end of the spike trains. This distance might be seen as a rate profile comparison in which a convolution kernel is used to smooth the rate profiles (Dayan and Abbot, 2001). Any other convolution kernel or another $L^{n}$ norm could be used. We will use $D_{\mathrm{R}^{*}}$ to refer to the van Rossum spike train distance calculated with a rectangular convolution kernel instead of the usual truncated exponential kernel. For the rectangular kernel we will take the parameter $\tau_{\mathrm{R}}$ to be the standard deviation of the kernel, like for the truncated exponential kernel. The dependence of $D_{\mathrm{R}}$ for the two extremes of $\tau_{\mathrm{R}}$ is independent of the kernel function used up to a constant factor. At one extreme, $D_{\mathrm{R}}\left[\tau_{\mathrm{R}} \rightarrow 0\right] \sim n_{x}+n_{y}$ is a coincidence detector. In the opposite limit $D_{\mathrm{R}}\left[\tau_{\mathrm{R}} \rightarrow \infty\right] \sim\left(n_{x}-n_{y}\right)^{2}$. In consequence, in contrast to $D_{\mathrm{V}}$, the van Rossum spike train distance $D_{\mathrm{R}}$ is not monotonic with $\tau_{\mathrm{R}}$, and the difference between the magnitude of the values at the two extremes (Table 1) is in general higher.

\subsubsection{Binning distance $D_{B}$}

The calculation of the mutual information with spike train distances in combination with a classifier has been motivated to avoid the use of binning in the estimation of the probabilities $p(\mathbf{R}, S)$ (Section 2.1). To check to which degree binning is inconvenient by itself or becomes problematic only when used to construct a high dimensional $\mathbf{R}$ space (e. g. Strong et al., 1998), we also study a spike train distance based on binning (Schnupp et al., 2006). For each spike train, the spikes are binned in $N_{B}$ bins of width $\tau_{\mathrm{B}}$. The distance $D_{\mathrm{B}}$ is calculated straightforwardly from the differences between the number of spikes in each bin for two spike trains. Like for $D_{\mathrm{R}}$, the sum of the squared differences is here used unless stated otherwise. We will use $D_{\mathrm{B}^{*}}$ to refer to the bins distance calculated by taking the absolute value of the difference instead of the squared difference. Accordingly, this distance is proportional to $D_{\mathrm{R}}$ in the two extremes of $\tau_{\mathrm{B}}$ (Table 1). For $\tau_{\mathrm{B}} \rightarrow 0$ the bins will contain at maximum one spike, and the full bins will generally not coincide for each spike train. In consequence, $D_{\mathrm{B}}\left[\tau_{\mathrm{B}}=0\right]=n_{x}+n_{y}$. Oppositely, if only one bin is used for the whole length $L$ of the spike trains, we get $D_{\mathrm{B}}\left[\tau_{\mathrm{B}}=L\right]=\left(n_{x}-n_{y}\right)^{2}$. The main difference with $D_{\mathrm{V}}$ and $D_{\mathrm{R}}$ is that the rate code is not only represented by $\tau=\infty$, but already by $\tau=L$, the length of the spike trains.

\subsubsection{Schreiber dissimilarity $D_{S}$}

For completeness we also discuss a dissimilarity measure derived from the correlation-based reliability measure of Schreiber et al. (2003). This measure has also occasionally be used in the discrimination analysis (Wang et al., 2007), although we will now show it does not not reflect different degrees of sensitivity to the temporal structure, going from a rate code to a coincidence code. At first 
the spike trains are also convolved with a convolution kernel resulting in the vectors $\vec{s}_{x, y}$ when the convolution is applied for a discrete number of sampling times. The time scale parameter $\tau_{\mathrm{S}}$ is related to the width of the kernel like for $D_{\mathrm{R}}$. The similarity measure is defined as the cross correlation

$$
S_{\text {corr }}=\frac{\vec{s}_{x} \cdot \vec{s}_{y}}{\left|\vec{s}_{x}\right|\left|\vec{s}_{y}\right|}
$$

A dissimilarity measure based on $S_{\text {corr }}$ is implemented as $D_{\mathrm{S}}=1-S_{\text {corr }}$. The measure $S_{\text {corr }}$ corresponds to an angle in the space of the spike trains (Paiva et al., 2009). Therefore, the dependence of $S_{\text {corr }}$ on $\tau_{\mathrm{S}}$ differs significantly from the one of $D_{\mathrm{R}}$ on $\tau_{\mathrm{R}}$ (Table 1). Again, this dependence is independent of the particular convolution kernel. The limit $D_{\mathrm{S}}\left[\tau_{\mathrm{S}} \rightarrow 0\right]$ is consistent with the ones obtained for $D_{\mathrm{V}}\left[\tau_{\mathrm{V}} \rightarrow 0\right]$ and $D_{\mathrm{R}}\left[\tau_{\mathrm{R}} \rightarrow 0\right]$, corresponding to the maximal sensitivity to spike timing. Assuming no exact coincidence of spikes, in this limit $\vec{s}_{x} \cdot \vec{s}_{y}=0$ and thus $D_{\mathrm{S}}=1$. By contrast, for $\tau_{\mathrm{S}} \rightarrow \infty$ the convoluted signals $\vec{s}_{x, y}$ will be both constant with a different value determined by the respective number of spikes in each train, $n_{x, y}$. However, due to the normalized dot product, $\frac{\vec{s}_{x}}{\left|\vec{s}_{x}\right|} \cdot \frac{\vec{s}_{y}}{\left|\vec{s}_{y}\right|}$ will always have the maximum value of 1 , so that $D_{\mathrm{S}}\left[\tau_{\mathrm{S}} \rightarrow \infty\right]=0$. In consequence, $D_{\mathrm{S}}\left[\tau_{\mathrm{S}} \rightarrow \infty\right]$ cannot be associated with a rate code, since it is not sensitive to the difference in the number of spikes. Accordingly, $D_{\mathrm{S}}$ does not fulfill the requirements to study the discriminative precision (Victor and Purpura, 1997) and we will not use it in the rest of this study.

\subsubsection{General remarks}

We finish the description of the measures with two general remarks. In first place, as already pointed out by Victor and Purpura (1997), for certain values of the parameters the spike train distances do not accomplish the conditions required to be a distance, in the strict sense. In particular, for $D_{\mathrm{V}, \mathrm{R}, \mathrm{B}}\left[\tau_{\mathrm{V}, \mathrm{R}, \mathrm{B}}=\infty\right]$, zero values are not only obtained for identical spike trains, but also for spike trains with equal number of spikes. Similarly, for the dissimilarity measure $D_{\mathrm{S}}\left[\tau_{\mathrm{S}} \rightarrow \infty\right]$, zero values are obtained for any pair of spike trains, and it is clearly not a distance. Being aware of these violations we still generally refer to $D_{\mathrm{V}, \mathrm{R}, \mathrm{B}}$ as spike train distances and consider that identity is redefined in these cases to the classes of spike trains leading to zero distance. In second place, there is no unique way to relate the parameter of the distances to a time scale of the spike trains. For $D_{\mathrm{V}}$ we have chosen $\tau_{\mathrm{V}}=\frac{2}{q}$. This selection has been used in several previous studies (Victor and Purpura, 1996; Reich et al., 2001b; Huetz et al., 2009), but it is also common to use $\tau_{\mathrm{V}}=\frac{1}{q}$ (e. g. Victor and Purpura, 1997; Samonds and Bonds, 2004; Huetz et al., 2006). Notice that, although for an individual pair of spikes 


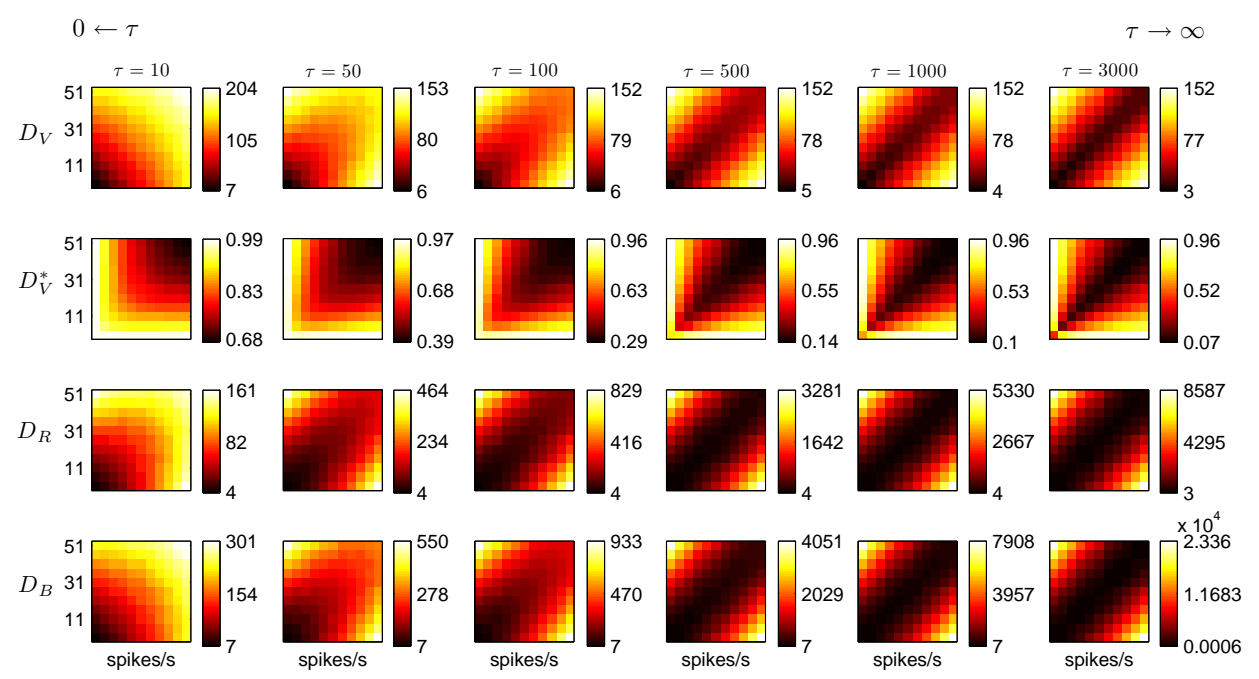

Figure 1: Measures of spike train dissimilarity in dependence on the rates of two time-independent Poisson spike trains. Each column corresponds to a fixed value of the time scale parameter $\tau(\mathrm{ms}) . M=50$ realizations of length $3 \mathrm{~s}$ were generated for each rate. Values shown are averages over all the possible pairs of spike trains for two given rates. Notice that the scale is set independently for each subplot.

$2 / q$ corresponds to the maximum time shift allowed, for complete spike trains $\tau_{\mathrm{V}}=2 / q$ does not indicate the time scale of the time shifts actually used in the minimal cost transformation. For $D_{\mathrm{R}}$ and $D_{\mathrm{S}}$ we take as the time scale parameter the standard deviation of the convolution kernel. An alternative criteria could be the decay time of the convolution kernel (Wang et al., 2007). For $D_{\mathrm{B}}$, the time scale parameter is the width of the bins. This arbitrariness introduces some ambiguity in the alignment of the parameter $\tau$ for different measures and the comparison of the obtained $\tau^{*}$. Furthermore, except for $D_{\mathrm{B}}$, the time scale parameter can take values higher than the length of the spike trains $L$ without being associated with a rate code.

\section{Results}

\subsection{Spike train distances for time-independent Poisson spike trains with distinct rates}

We first examine the spike train distances for pairs of Poisson spike trains with distinct time-independent rates $\lambda_{x}, \lambda_{y}$. We consider as an a priori condition of 
consistency that the lowest distance to spike trains generated with rate $\lambda_{x}$ should be obtained for $\lambda_{y}=\lambda_{x}$. In Figure 1 we show the results obtained for $D_{\mathrm{V}}, D_{\mathrm{V}}^{*}$, $D_{\mathrm{R}}$, and $D_{\mathrm{B}}$. We see that $D_{\mathrm{V}}, D_{\mathrm{R}}$, and $D_{\mathrm{B}}$ have a similar behavior. From high to low $\tau$ the consistency is gradually lost. This loss can be understood from the lack of normalization of these spike train distances. The maximum possible value of the distances depends on $\lambda_{x}$ and $\lambda_{y}$, that determine the total number of spikes $n=n_{x}+n_{y}$. In particular, for a fixed $\lambda_{x}$, a lower $\lambda_{y}$ results in a lower maximum possible value. For example, for $D_{\mathrm{V}}$, when $\tau$ approaches the time scale indicated by $\frac{1}{\lambda_{x, y}}$, the effect of the different maximum possible values corresponding to different $n$ becomes progressively more relevant, since insertions and deletions dominate over shifts. In consequence, for small $\tau$ the lowest distances to spike trains generated with a rate $\lambda_{x}$ are not obtained for $\lambda_{y}=\lambda_{x}$ anymore, but for $\lambda_{y} \ll \lambda_{x}$.

The loss of consistency of $D_{\mathrm{V}}, D_{\mathrm{R}}$, and $D_{\mathrm{B}}$ also results in a worse discrimination between the stimuli whose responses are Poisson spike trains with different rates. For example, for $\tau=10 \mathrm{~ms}$, a classifier using any of these three metrics assigns all the spike trains to the stimulus eliciting the lowest rate. The main difference between these three distances is the dependence of their magnitudes on $\tau$. As discussed in Section 2.2, this is due to the specific functionality on the sum or the difference of total spikes in the limit of small or high $\tau$, respectively (Table 1).

In contrast to $D_{\mathrm{V}}$, in the limit of small $\tau_{\mathrm{V}}$, low values of $D_{\mathrm{V}}^{*}$ occur for high rates. This indicates that although the absolute transformation cost increases with the rates, the cost normalized by the maximum possible cost is indeed lower. For $\tau=10 \mathrm{~ms}$ we see that the range of values decreases, and approaches 1 . Since $D_{\mathrm{V}}^{*}\left[\tau_{\mathrm{V}} \rightarrow 0\right] \rightarrow 1$, towards this limit the distance progressively will loose all its specificity. However, how this affects the classification will depend on wether the distances resulting from the same or different stimuli are still separable. For $\tau_{\mathrm{V}} \rightarrow \infty$ the consistency condition is fulfilled, but due to the normalization the distance does not only depend on the difference between the rates (Table 1).

These results confirm that the measures cover the range of time scales sensitivity going from a coincidence detector to a rate code distance. However, each measure has particular magnitudes and dependencies on the rates, and therefore may lead to a different discrimination of the stimuli associated to the rates.

\subsection{Information and discriminative precision for time-dependent Poisson spike trains}

\subsubsection{Dependence on the measure and the classifier}

We here examine the dependence of $I^{*}\left(S, S^{P} ; \tau\right)$ and $\tau^{*}$ on the measure and on the classifier. We simulate time-dependent Poisson spike trains in which the only source of temporal structure is the modulation of the rates. We compose the spike trains by concatenation of several intervals of length $L_{i}$, where $i=1, \ldots, N_{L}$. For 
each interval time-independent Poisson processes are generated. Furthermore, only two stimuli $S_{x}$ and $S_{y}$ are considered, so that the spike trains are fully described by the rates $\left(\lambda_{x, i}, \lambda_{y, i}\right)$.

In Figure 2 we show the dependence of $I^{*}\left(S^{P}, S ; \tau\right)$ on the time scale $\tau$ for four examples with different configurations of the time-dependent rates. For all examples A-D we fix $N_{L}=4$. In example A the rates on each interval for the two stimuli are such that the stimuli can be well discriminated by the total rates. In example $\mathrm{B}$ the total rates still differ but are closer to each other. In examples $\mathrm{C}$ and $\mathrm{D}$ the total rates are equal, and only the local rates in each interval $L_{i}$ differ. These local differences in the rate are higher for example $\mathrm{C}$. We show the results for four different classifiers corresponding to different values of the exponent $z$ (Equation 2).

We see that $I_{\max }^{*}\left(S^{P}, S\right)$ does not critically depend on the measure. In particular, the values of $I_{\max }^{*}\left(S^{P}, S\right)$ obtained by $D_{\mathrm{B}}$ are not lower than those obtained by the other measures. This indicates that, for these examples, this distance based on binning is as valid as the other more elaborated measures. While $I_{\max }^{*}\left(S^{P}, S\right)$ is almost the same for different measures, the shape of $I^{*}\left(S^{P}, S ; \tau\right)$ varies considerably across them. In the first place, for $\tau_{\mathrm{B}}$ equal the length $L$ of the spike trains, $D_{\mathrm{B}}$ already corresponds to a rate code distance. By contrast, the other measures are still sensitive to the temporal structure as long as $\tau<\infty$. Therefore, for the examples $\mathrm{C}$ and $\mathrm{D}$, in which the total rate is the same for the responses to $S_{x}$ and $S_{y}$ and a rate code cannot discriminate between them, the other measures still result in high values of $I^{*}\left(S^{P}, S ; \tau\right)$ for time scales higher than L. On the other limit, for $\tau \rightarrow 0$, the expected decrease of the mutual information depends on the loss of consistency as discussed in Section 3.1. In general, $I^{*}\left(S^{P}, S ; \tau\right)$ decreases more slowly for $D_{\mathrm{V}}^{*}$ than for the rest of the measures. This is due to the normalization and the effect of the maximum possible value of the distance on the loss of consistency, as described in Section 3.1.

Since the distances depend smoothly on $\tau$, also $I^{*}\left(S^{P}, S ; \tau\right)$ is expected to be smooth if $\tau$ is sufficiently sampled. The flatness of the peak depends on several factors. If high values of $I^{*}\left(S^{P}, S ; \tau\right)$ are obtained like in examples A and C, the peak is flatter due to the saturation of the sensitivity of $I^{*}\left(S^{P}, S ; \tau\right)$. For these examples, since the stimuli are very different, they are more likely to be discriminated better at any time scale. Furthermore, the flatness depends on the sensitivity of each measure at each time scale. The most obvious example is that the sensitivity of $D_{\mathrm{V}}, D_{\mathrm{V}}^{*}$, and $D_{\mathrm{R}}$ to temporal structure for $\tau>L$ will result in a flatter peak towards high time scales $\tau$. In the opposite limit $\tau \rightarrow 0$, the initial decrease from $I_{\text {max }}^{*}\left(S^{P}, S\right)$ is slower for $D_{\mathrm{R}}$. This occurs because for $D_{\mathrm{V}}$ and $D_{\mathrm{B}}$ the resolution cannot be further increased when $\tau$ is sufficiently small. This saturation of the resolution for small $\tau$ is caused by the binning in $D_{\mathrm{B}}$ and by the maximal cost of 2 in 

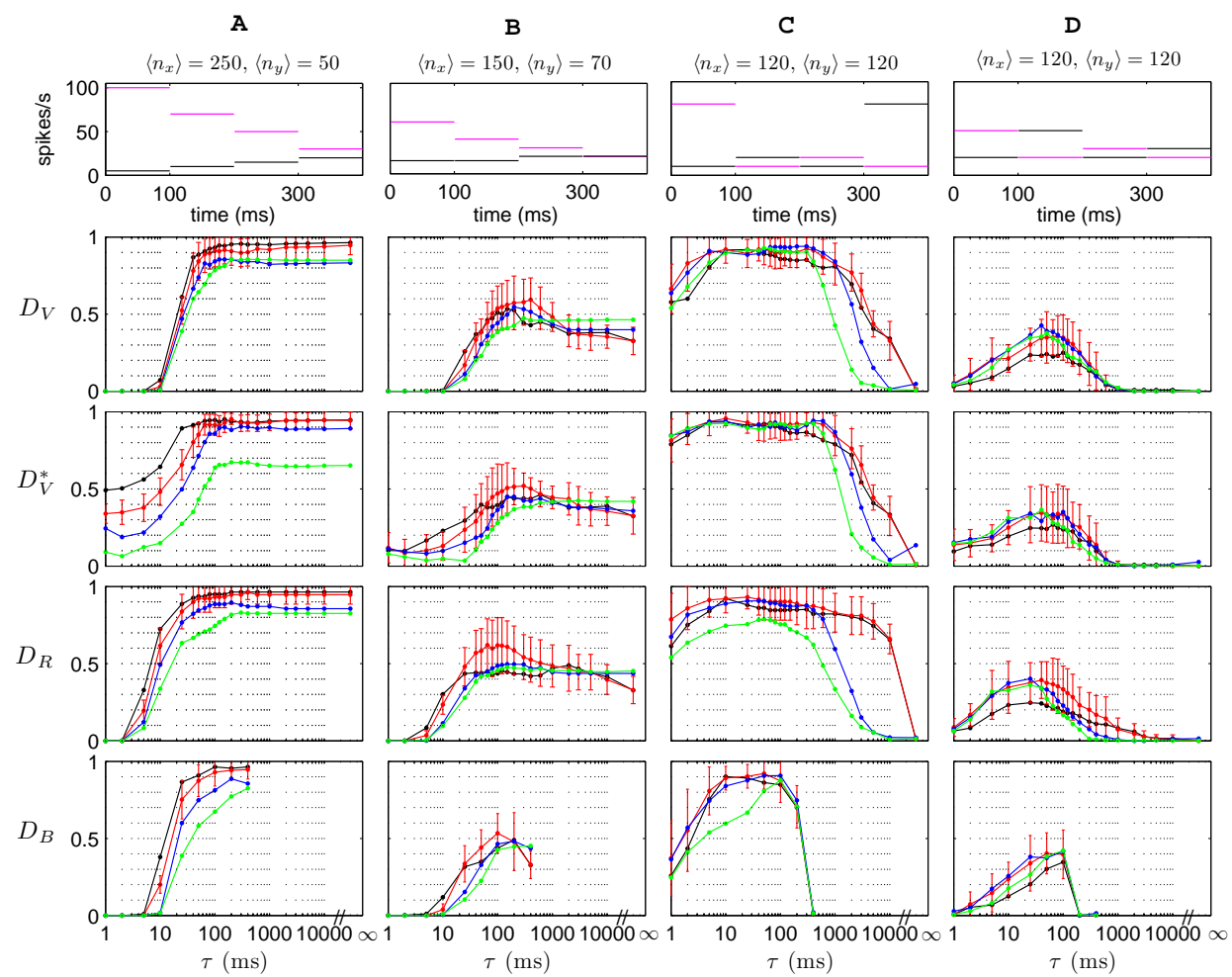

Figure 2: Dependence of $I^{*}\left(S^{P}, S ; \tau\right)$ on the measure and classifier for simulated time-dependent Poisson spike trains. The different columns correspond to examples A-D, with different combinations of modulated rates, as shown in the first row. In all the cases the responses to two stimuli $S_{x}$, $S_{y}$ are simulated as Poisson processes with piecewise constant modulated rates. For all the examples $N_{L}=4$ intervals of length $100 \mathrm{~ms}$ are simulated with constant rates $\left[\lambda_{x, 1}, \lambda_{y, 1} ; \lambda_{x, 2}, \lambda_{y, 2} ; \lambda_{x, 3}, \lambda_{y, 3} ; \lambda_{x, 4}, \lambda_{y, 4}\right]$ (spikes/s). A: 100, $5 ; 70,10 ; 50,15 ; 30,20$ spikes/s. B: 60,$15 ; 40,15 ; 30,20 ; 20,20$ spikes/s. C: 80,$10 ; 10,20 ; 20,10 ; 10,80$ spikes/s. D: 50,$20 ; 20,50 ; 30,20 ; 20,30$ spikes/s. We indicate the total average spike counts $\left\langle n_{x}\right\rangle,\left\langle n_{y}\right\rangle$. Four classifiers with different exponent (Equation 2) are used: $z=-8$ (black), $z=-2$ (red), $z=2$ (blue), and $z=8$ (green). Averages across $K=20$ independent realizations with $M=20$ trials per stimulus are shown. The standard deviations across the $K$ realizations are displayed only for $z=-2$. 


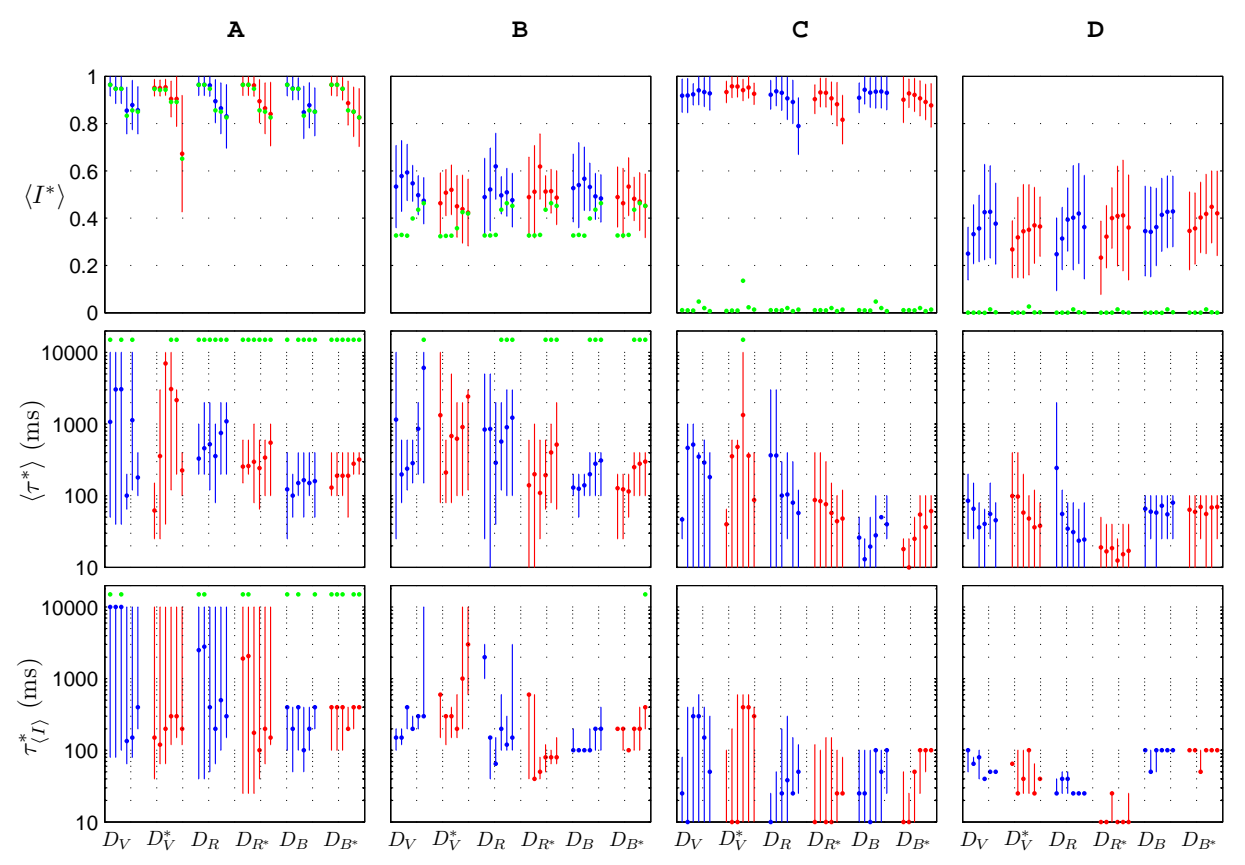

Figure 3: Maximal mutual information $I_{\max }^{*}\left(S^{P}, S\right)$ and optimal time scale $\tau^{*}$ in dependence on the measure and classifier for the simulated examples shown in Figure 2. Each column shows the results for the corresponding simulations A-D in Figure 2. For each measure the results are shown for exponents $z=$ $[-8,-4,-2,2,4,8]$, from left to right, and alternate blue and red dots are used to distinguish the results of adjacent measures. In the first row, we also show with green dots the mutual information obtained with a rate code, $I_{\text {count }}^{*}\left(S^{P}, S\right)$. In the second and third row we indicate with green dots when $\tau=\infty$ was optimal for some realization. Details on the calculation of the averages and the error bars are provided in Section 2.1. 
$D_{\mathrm{V}}$.

The exponent $z$ of the classifier also results in significant changes in the shape of $I^{*}\left(S^{P}, S ; \tau\right)$. Actually, $z$ influences $I_{\max }^{*}\left(S^{P}, S\right)$ more than the measure. To see more in detail the dependence of $I_{\max }^{*}\left(S^{P}, S\right)$ and $\tau^{*}$ on the measures and exponents $z$, we examine in Figure 3 the values extracted from the shapes of $I^{*}\left(S^{P}, S ; \tau\right)$ shown in Figure 2. For example A, negative exponents are preferred, and for example $D$ the positive ones. For examples $B$ and $C$ small $|z|$ lead to higher $I_{\max }^{*}\left(S^{P}, S\right)$. The particular dependence on $z$ changes across measures, but is qualitatively similar indicating that $z$ is associated with the geometry resulting from the distances. The variability of $I_{\max }^{*}\left(S^{P}, S\right)$, quantified by the standard deviation across trials, does not depend crucially on the measure or exponent.

The optimal time scale $\left\langle\tau^{*}\right\rangle$ is much more variable across measures. Furthermore, although some trends are observed in dependence on the exponent, the clarity and shape of this dependence change from measure to measure much more than for the dependence of $I_{\max }^{*}\left(S^{P}, S\right)$. The broad error bars indicate the high variability of $\tau^{*}$ for different realizations of the analysis (Section 2.1). The same qualitative conclusions regarding the high variability are obtained for $\tau_{\langle I\rangle}^{*}$. Here the error bars quantify the flatness of the peak, showing that in general $D_{\mathrm{B}}$ leads to a sharper peak. Comparing across examples, we see that higher values of $\tau^{*}$ are obtained for examples $\mathrm{A}$ and $\mathrm{B}$, in which the responses to the two stimuli have different total rates. For example A, values of $\tau^{*}=\infty$ occur. For examples $\mathrm{C}$ and $\mathrm{D}, \tau^{*}$ is lower and barely related to the time scale of the intervals $\left(L_{i}=100 \mathrm{~ms}\right)$. In all these examples this time scale is kept fixed, and the changes in $\tau^{*}$ result from the local balance of the different rates in response to the two stimuli. Furthermore, comparing $D_{\mathrm{R}}$ and $D_{\mathrm{R}^{*}}$, notice that $\tau^{*}$ does not only depend on the distance used but also on the kernel. The rectangular convolution kernel, which is symmetric with respect to the spike time, generally gives the lowest values of $\tau^{*}$. This is because it is effectively wider than the truncated exponential convolution kernel.

Besides determining $\tau^{*}$, to assess the relevance of temporal coding in the discrimination of the stimuli, we need to compare $I_{\text {max }}^{*}\left(S^{P}, S\right)$ and $I_{\text {count }}^{*}\left(S^{P}, S\right)$. For examples $\mathrm{C}$ and $\mathrm{D}$, in which the total rates are equal, $I_{\text {count }}^{*}\left(S^{P}, S\right) \simeq 0$ independently of the measure and exponent. For examples A and $\mathrm{B}, I_{\text {count }}^{*}\left(S^{P}, S\right)$ depends more on $z$ than on the measure. Accordingly, the index of temporal coding $\Theta$ (Equation 6) varies substantially when $I_{\text {max }}^{*}\left(S^{P}, S\right)$ and $I_{\text {count }}^{*}\left(S^{P}, S\right)$ calculated with the same $z$ are used to calculate it. In particular, for example B, while for positive exponents the role of temporal coding is almost irrelevant, for negative exponents $\Theta>0.5$. Considering the variability observed for $\tau^{*}$ and for the difference between $I_{\text {max }}^{*}\left(S^{P}, S\right)$ and $I_{\text {count }}^{*}\left(S^{P}, S\right)$, we see that the discriminative precision and the role of temporal coding are more difficult to evaluate than the mutual information between stimuli and predicted stimuli. 


\subsubsection{Dependence on the length of the spike trains for different simulated codes}

In the examples above we have not made any assumption about the cause of the modulation. The modulation can represent the effect of short-term adaptation in the transient responses to the onset of a constant stimulus, or the effect of correlations with a dynamic stimulus. In the first case the selection of a given length $L$ of the spike trains for the discrimination analysis involves some assumption about the time during which the constant stimuli are encoded. To further study the relation of the discriminative precision with the properties of the code, we examine how it depends on the length $L$.

For transient responses, latency has been shown to contain a high amount of information about the stimuli (e. g. Reich et al., 2001b; Panzeri et al., 2001). To model this effect in Figure 4 we consider two constant stimuli $S_{x}$ and $S_{y}$ that elicit, during a short fixed interval, the same elevation of the rate with respect to a baseline level, but with different delays with respect to the onset time. Furthermore, after this transient response, a constant rate is associated to each stimulus. In a first example (E) these after-transient rates are equal, while for a second example (F) the rate is higher for the stimulus with the larger latency. This difference in the after-transient rates, depending on the selected length $L$, suffices to produce a rate code discrimination. Here we only show the results for $D_{\mathrm{V}}$, but the conclusions about the estimation and interpretation of the mutual information and discriminative precision are qualitatively similar for the other measures.

We examine how $I_{\max }^{*}\left(S^{P}, S\right)$ and $\tau^{*}$ depend on the latency difference $L_{2}$ and on the after-transient interval length $L_{4}$. In example $\mathrm{E}$ the after-transient rates are equal, leading to $I_{\text {count }}^{*}\left(S^{P}, S\right) \simeq 0$ independently of $L_{2}$ and $L_{4}$. Since there is no overlap of the periods of elevated rate and the increase of the rates is high with respect to the baseline rate, high values of $I_{\max }^{*}\left(S^{P}, S\right)$ are obtained independently of the latency difference. In this example, the after-transient interval length $L_{4}$ does not affect the amount of information. Therefore, $\Theta$ is also similar for different latency differences and after-transient interval length $L_{4}$, indicating a constant contribution of temporal coding to discrimination. The optimal time scales $\left\langle\tau^{*}\right\rangle$ and $\tau_{\langle I\rangle}^{*}$, while almost independent of $L_{4}$, increase consistently in dependence on the latency difference $L_{2}$. Accordingly, the discriminative precision depends only on $L_{2}$.

For example $\mathrm{F}$, for which the after-transient rates differ, we get different results. For small $L_{4}$ the dependence on $L_{2}$ resembles the one found in example $\mathrm{E}$ as expected, since the total number of spikes is still similar for responses to both stimuli. When $L_{4}$ increases $I_{\text {count }}^{*}\left(S^{P}, S\right)>0$ is obtained indicating that a rate code is enough to discriminate. The length $L_{4}$ necessary to obtain $I_{\text {count }}^{*}\left(S^{P}, S\right)>$ 0 depends on the latency difference $L_{2}$. This is because during the latency interval 

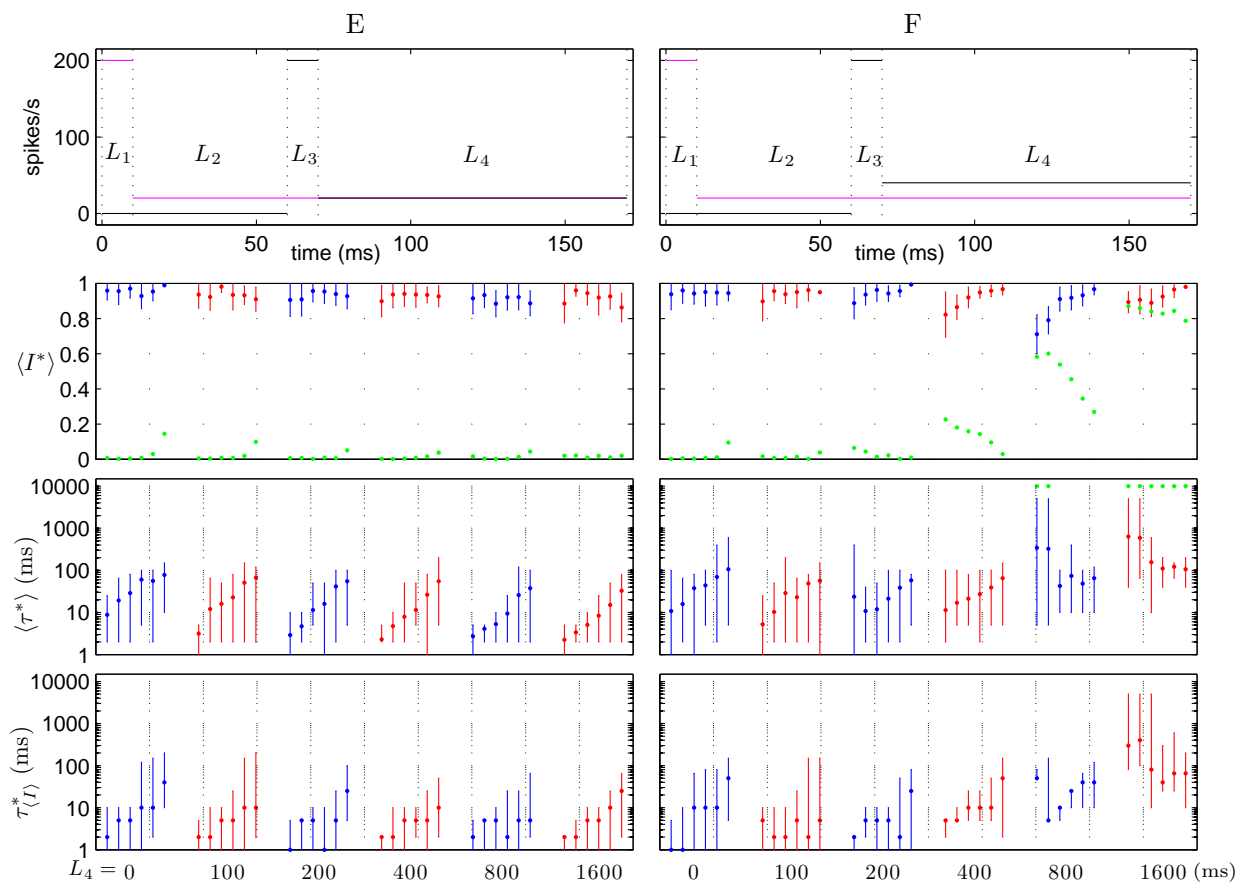

Figure 4: Dependence of $I_{\max }^{*}\left(S^{P}, S\right)$ and $\tau^{*}$ on the latency difference $L_{2}$ and the length of the after-transient interval $L_{4}$, for spike trains simulating the response to transient constant stimuli. Four intervals with constant rates for each stimulus are generated as shown in the first row. $L_{1}=10 \mathrm{~ms}$ models the transient response of stimulus $S_{x} . L_{2}=[0,10,25,50,100,200]$ ms models the latency difference. $L_{3}=10 \mathrm{~ms}$ corresponds to the time of the transient response of $S_{y}$. $L_{4}=[0,100,200,400,800,1600] \mathrm{ms}$ is the after-transient interval. Following the notation in Figure 2, the rates are: E: [200, 0;20, 0;20,200; 20,20] spikes/s, F: $[200,0 ; 20,0 ; 20,200 ; 20,40]$ spikes/s. In the first row the modulated rates are shown using $L_{2}=50 \mathrm{~ms}$ and $L_{4}=100 \mathrm{~ms}$ for exemplary purpose. In example $\mathrm{E}$ the rates are the same during the after-transient interval. In the other rows, for each length $L_{4}$ the results are shown for all the lengths $L_{2}$ with the same color, analogously to Figure 3. Results are shown for $D_{\mathrm{V}}$ with $z=-2, K=20$, and $M=20$. 
the stimulus with the earlier response has a higher rate that has to be balanced in the after-transient interval by the higher rate of the other stimulus. On the other hand, $I_{\max }^{*}\left(S^{P}, S\right)$ increases with the latency difference $L_{2}$ because information can be obtained from both the latency and rate difference if temporal coding is used. Since both $I_{\text {count }}^{*}\left(S^{P}, S\right)$ and $I_{\text {max }}^{*}\left(S^{P}, S\right)$ change with $L_{2}$ and $L_{4}$, so does $\Theta$. In this example there are two different time scales at which the stimuli can be discriminated, and one or the other is more relevant depending on the balance between the latency difference and $L_{4}$. For intermediate values of $L_{4}, I_{\max }^{*}\left(S^{P}, S\right)$ decreases for small latency differences $L_{2}$, showing that there is no optimal time scale at which the two sources of information can be combined. The optimal time scale $\tau^{*}$ does not mainly depend on the latency difference as in example E. For sufficiently large $L_{4}$, both $\left\langle\tau^{*}\right\rangle$ and $\tau_{\langle I\rangle}^{*}$ increase with $L_{4}$ indicating that a rate code is enough for discrimination. For small $L_{4}$ the dependence on the latency difference $L_{2}$ is still visible, in particular for $\left\langle\tau^{*}\right\rangle$. Altogether, we see that when different sources of information exist that are not on the same time scale, it is difficult to interpret $\tau^{*}$ as something more than the parameter for which the best discrimination is obtained.

We also consider another possible encoding of the transient presentation of constant stimuli (Figure 5). In this case a phasic response is elicited with a different elevation of the rate for each stimulus $S_{x}, S_{y}$. Furthermore, we assume that after this increase the rate returns to the same baseline level independently of the stimulus. For a fixed interval of the transient response $L_{1}$ and fixed values of the rates during the transient response to each stimulus, we vary the after-transient interval and thereby the length of the spike trains used for the discrimination analysis $L$.

We examine the dependence of $I_{\max }^{*}\left(S^{P}, S\right)$ and $\tau^{*}$ on $L$ and on the exponent of the classifier. Like for example $\mathrm{E}$, the after-transient rates do not add any information about the stimuli. Therefore, we expect that also here $I_{\max }^{*}\left(S^{P}, S\right)$ is independent of $L$. However, if we restrict ourselves to a negative exponent $z=-2$ as in Figure 4, $I_{\max }^{*}\left(S^{P}, S\right)$ increases with $L$. Since there is no extra information in $L_{2}$, this increase can only be caused by a change in the ability of the classifier to extract the existent information in each case. As discussed in Section 2.1, $I_{\max }^{*}\left(S^{P}, S\right)$ is a lower bound of the total information $I(\mathbf{R}, S)$. To have a reference of the total information in the responses, we show an estimate of $I(\mathbf{R}, S)$ according to Equation 1. We use only the number of spikes in the first interval to represent $\mathbf{R}$, since there is no information about the identity of the stimulus in $L_{2}$. This estimate is calculated independently using a larger number of trials per stimulus and checking for its convergence to avoid any upward bias. If we compare for different $L_{2}$ the maximal $I_{\max }^{*}\left(S^{P}, S\right)$ across $z$, we see that this maximum remains almost constant for different $L_{2}$. Oppositely, $I_{\text {count }}^{*}\left(S^{P}, S\right)$ decreases with $L_{2}$ even if the 
optimal $z$ is chosen independently from the optimal $z$ for $I_{\max }^{*}\left(S^{P}, S\right)$. This is because for Poisson processes the variance is equal to the mean, and thus it is harder to use the different rates in $L_{1}$ to discriminate when $L_{2}$ increases and the number of spikes in this interval differs more. Therefore, $\Theta$ is more sensitive to the selection of $L_{2}$ than $I_{\max }^{*}\left(S^{P}, S\right)$. This variability also affects the discriminative precision. The optimal time scale estimated as the average across realizations $\left\langle\tau^{*}\right\rangle$ results in higher values than $\tau_{\langle I\rangle}^{*}$, calculated from the average shape of $I^{*}\left(S^{P}, S ; \tau\right)$. The large error bars of $\left\langle\tau^{*}\right\rangle$ indicate a high variability across single realizations. From $\tau_{\langle I\rangle}^{*}$ we see that the peak is sharper for high $L_{2}$. Furthermore, for the exponents for which the maximal $I_{\max }^{*}\left(S^{P}, S\right)$ is obtained, $\tau^{*}$ is almost constant independently of $L_{2}$ but it does not correspond to the length of $L_{1}$.

\subsection{Information and discriminative precision for experimental responses to tran- sient constant stimuli}

For the simulated examples above we showed that the discrimination performance, the discrimination precision, and the relevance of temporal coding depend on the measure and classifier as well as on the length of the spike trains under consideration. We illustrated these dependencies for time-dependent Poisson spike trains and assuming several simple neural codes. To further examine what we can learn about the neural code with the discrimination analysis using the time scale parametric spike train distances we now study some real neural responses. In particular we analyze the responses of exemplary rat gustatory cells and one monkey visual cell available online at http://neurodatabase.org (Gardner, 2004). Di Lorenzo and Victor (2003) analyzed the reliability of mean rate response across trials in the nucleus of the solitary tract (NTS) of the rat for four taste quality stimuli: $\mathrm{NaCl}$, sucrose, quinine- $\mathrm{HCl}$, and $\mathrm{HCl}$. The authors applied discrimination analysis with the Victor distance to examine the potential contribution of temporal coding to the discrimination of the stimuli. The analysis was carried out for the total length of the recordings $(10 \mathrm{~s})$ as well as for only the interval related to the phasic response (the first $2 \mathrm{~s}$ ). We here analyzed three single neurons labeled 11, 9, and 4, to which we will refer as gustatory cells $g_{1}, g_{2}$, and $g_{3}$, respectively. For these cells 16,23 , and 19 trials for each stimulus were recorded respectively. In Figure 6 A-C we show the time-dependent rates averaged across trials for each stimuli. Notice the initial phasic response which lasts for the first two seconds approximately.

Aronov et al. (2003) studied the discriminability of transient presentations of stationary gratings of spatial phases in single neurons from the primary visual cortex of anesthetized macaque monkeys. The authors applied the discrimination analysis with the Victor distance to analyze the encoding of spatial phases. We here use one exemplary cell labeled $410106 t$, to which we will refer as $v_{1}$. Data comprise 

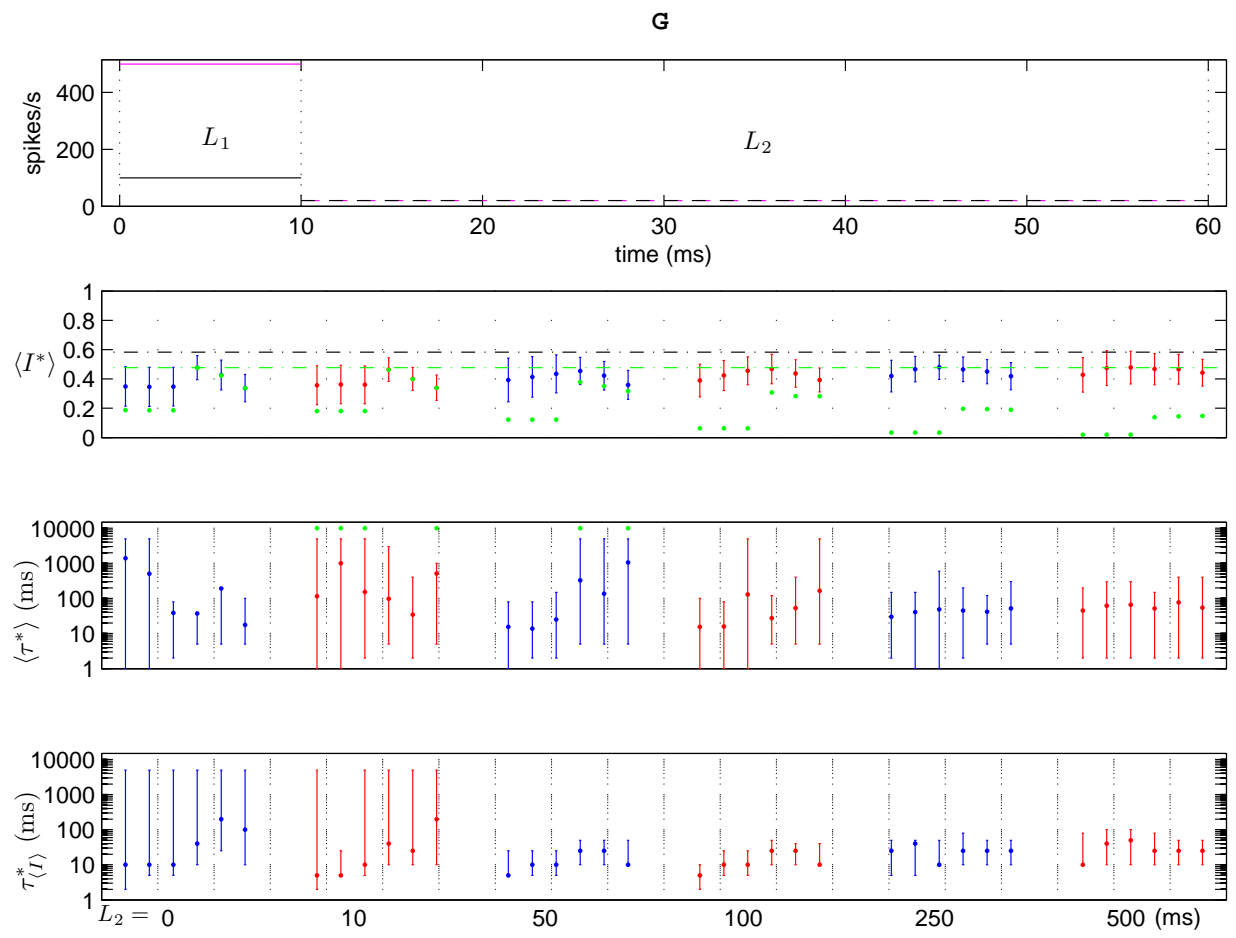

Figure 5: Dependence of $I_{\max }^{*}\left(S^{P}, S\right)$ and $\tau^{*}$ on the length of the after-transient interval and on the classifier for phasic rate responses. Two intervals with constant rates for each stimulus are generated, as shown in the first row. The first interval $L_{1}=10 \mathrm{~ms}$ models the phasic responses, while the second, $L_{2}=$ $[0,10,50,100,250,500] \mathrm{ms}$, is the after-transient interval. The rates, following the notation of Figure 2, are $[500,100 ; 20,20]$ spikes/s. The structure of the figure is analogous to Figure 3. For each length $L_{2}$, results for exponents $z=[-8,-4,-2,2,4,8]$ are shown with the same color. For the mutual information, we also show the maximal $I_{\max }^{*}\left(S^{P}, S\right)$ across all the exponents for $L_{2}=0$ (dotted green line), and the estimate (Equation 1) of $I^{*}(R, S)$ for $L_{2}=0$ (dotted black line). Results are shown for $D_{\mathrm{V}}, K=20$, and $M=20$. 

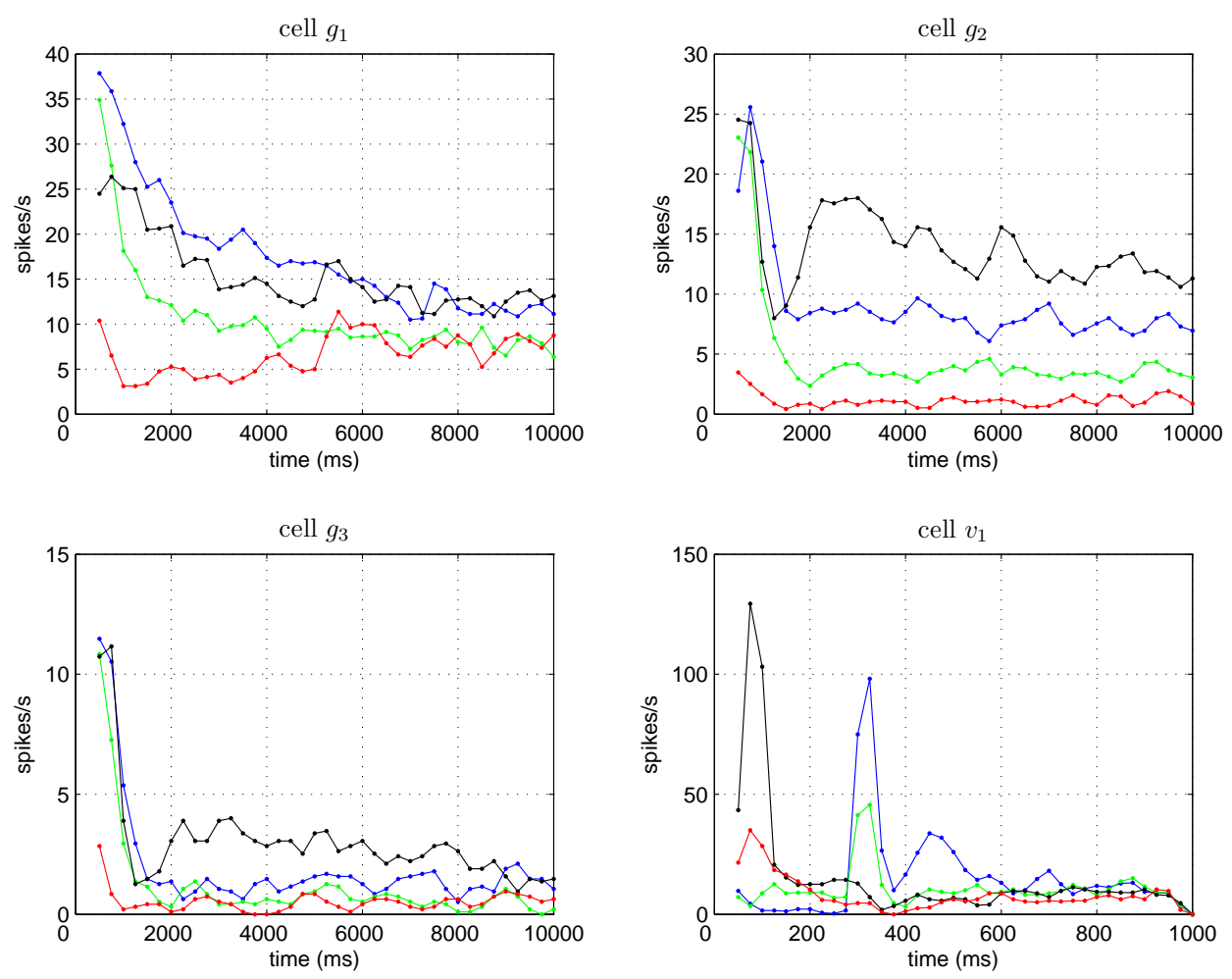

Figure 6: Time-dependent average rates for each stimulus of the four exemplary recorded cells responding to transient constant stimuli. For the gustatory cells $g_{1}$ $g_{3}$ the rates were calculated for a moving window of $500 \mathrm{~ms}$ with a step of 250 ms. For the visual cell $v_{1}$ the moving window was of $50 \mathrm{~ms}$ and a step of $25 \mathrm{~ms}$. Different colors correspond to different stimuli. 
the responses to 64 trials for four spatial phases (0, 90, 180, and 270 degrees). Each spike train corresponds to $236 \mathrm{~ms}$ of stimulus presentation plus $710 \mathrm{~ms}$ of a uniform field at the mean luminance. Accordingly, the length of the spike trains is $\sim 1 \mathrm{~s}$, which is one order of magnitude smaller than for the gustatory cells, as can be seen in Figure 6. The major peaks in the rate reflect the onset and offset of the stimuli.

\subsubsection{Dependence on the measure and the classifier}

We proceed in analogy to Section 3.2.2 and examine $I^{*}\left(S^{P}, S ; \tau\right)$ obtained with different measures and different exponents $z$ for $L$ equal to the whole length of the spike trains. In Figure 7 we see that, for the gustatory cells $g_{1-3}$, the shape of $I^{*}\left(S^{P}, S ; \tau\right)$ varies less for different exponents than for the visual cell $v_{1}$. Like for the simulated examples the normalization for $D_{\mathrm{V}}^{*}$ can lead to nonzero values for small time scales $\tau$.

In Figure 8 we show in more detail the dependence of $I_{\text {max }}^{*}\left(S^{P}, S\right)$ and $\tau^{*}$. Like for the simulations using Poissonian spike trains (Section 3.2.1), $I_{\text {max }}^{*}\left(S^{P}, S\right)$ depends more on the classifier than on the measure. In particular, $D_{\mathrm{B}}$ can discriminate equally well as the measures without binning. The highest values of $I_{\text {max }}^{*}\left(S^{P}, S\right)$ are generally obtained for the classifiers using low $|z|$ and negative exponents are preferred. Oppositely, for $I_{\text {count }}^{*}\left(S^{P}, S\right)$, positive exponents lead to higher values. In consequence, $\Theta$ is more sensitive to the classifier. To minimize this effect $I_{\text {max }}^{*}\left(S^{P}, S\right)$ and $I_{\text {count }}^{*}\left(S^{P}, S\right)$ should be optimized separately across $z$ before calculating $\Theta$. In contrast to the simulated examples, we here cannot generate additional trials to get an unbiased estimation of $I^{*}(\mathbf{R}, S)$ that takes the temporal structure into account. Therefore, we reduce $\mathbf{R}$ to the total rates to compare with the information $I^{*}(R, S)$ that can be obtained considering only a rate code $(\tau \rightarrow \infty)$. We use Equation 1 to calculate $I^{*}(R, S)$ and apply an analytical first order correction to reduce the bias (Treves and Panzeri, 1995). Notice that $I_{\max }^{*}\left(S^{P}, S\right)$ is not a lower bound for $I^{*}(R, S)$, as can be seen for those cells in which temporal coding is relevant. Nonetheless, $I^{*}(R, S)$ is an upper bound for $I_{\text {count }}^{*}\left(S^{P}, S\right)$ and thus it can be useful to quantify $\Theta$ when, like for cell $g_{1}$, $I_{\text {count }}^{*}\left(S^{P}, S\right)$ does not attain $I^{*}(R, S)$. Regarding $\tau^{*}$, we see that the values of $\left\langle\tau^{*}\right\rangle$ and $\tau_{\langle I\rangle}^{*}$ for the exponent leading to the optimal $I_{\max }^{*}\left(S^{P}, S\right)$ can differ in an order of magnitude across measures. In particular lower values are generally obtained for $D_{\mathrm{R}}$, especially when the symmetric rectangular convolution kernel is used. There is also a high variability across bootstrapping realizations, like for the simulated examples in Figure 3. Overall these results are consistent with the ones found for examples A-D of simulated data. The mutual information depends more on the classifier than on the measure, especially for the visual cell. This also holds for the index of temporal coding, which furthermore has a higher variability across 

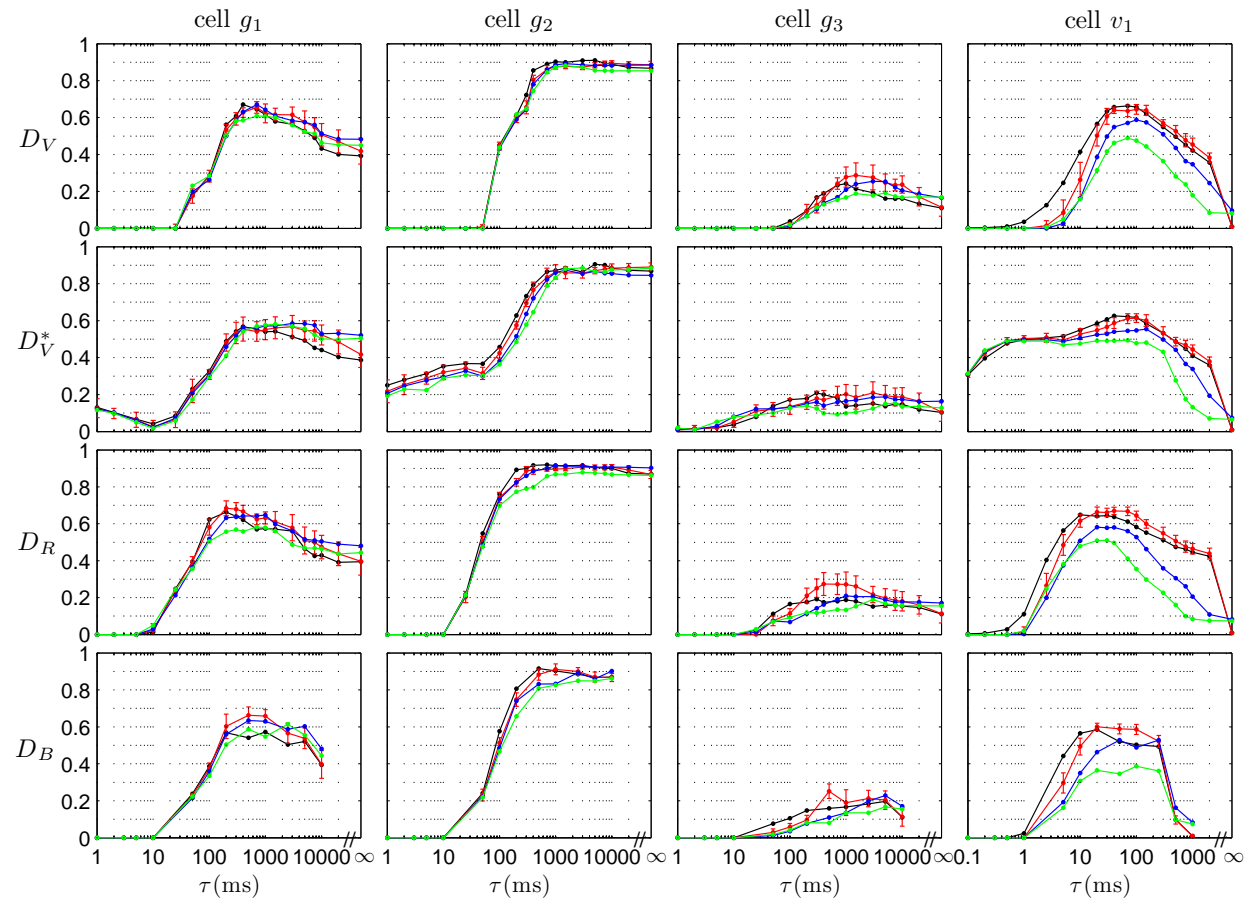

Figure 7: Dependence of $I^{*}\left(S^{P}, S ; \tau\right)$ on the measure and classifier for the four exemplary cells. The figure is analogous to Figure 2. Four classifiers with different exponent (Equation 2) are used: $z=-8$ (black), $z=-2$ (red), $z=2$ (blue), and $z=8$ (green). Results shown are averages across $K=20$ bootstrapping realizations in which $M^{\prime}=\frac{3}{4} M$ randomly chosen trials are used. The standard deviations of the mutual information across the $K$ bootstrapping realizations are displayed for $z=-2$.

classifiers given that not only $I_{\text {max }}^{*}\left(S^{P}, S\right)$ but also $I_{\text {count }}^{*}\left(S^{P}, S\right)$ depends on the classifier. The optimal time scale is significantly sensitive to both the measure and the classifier.

\subsubsection{Temporal accumulation of information}

The dependence of the results on the length of the spike trains $L$ used for the discrimination analysis was studied for the gustatory cells in Di Lorenzo and Victor (2003). In particular, the results obtained applying the discrimination analysis for the whole $10 \mathrm{~s}$ of the recordings were compared with those obtained for the first 2 $\mathrm{s}$, associated with the phasic response. This comparison revealed that the temporal coding was more relevant during the phasic response. Similarly, Victor and Pur- 

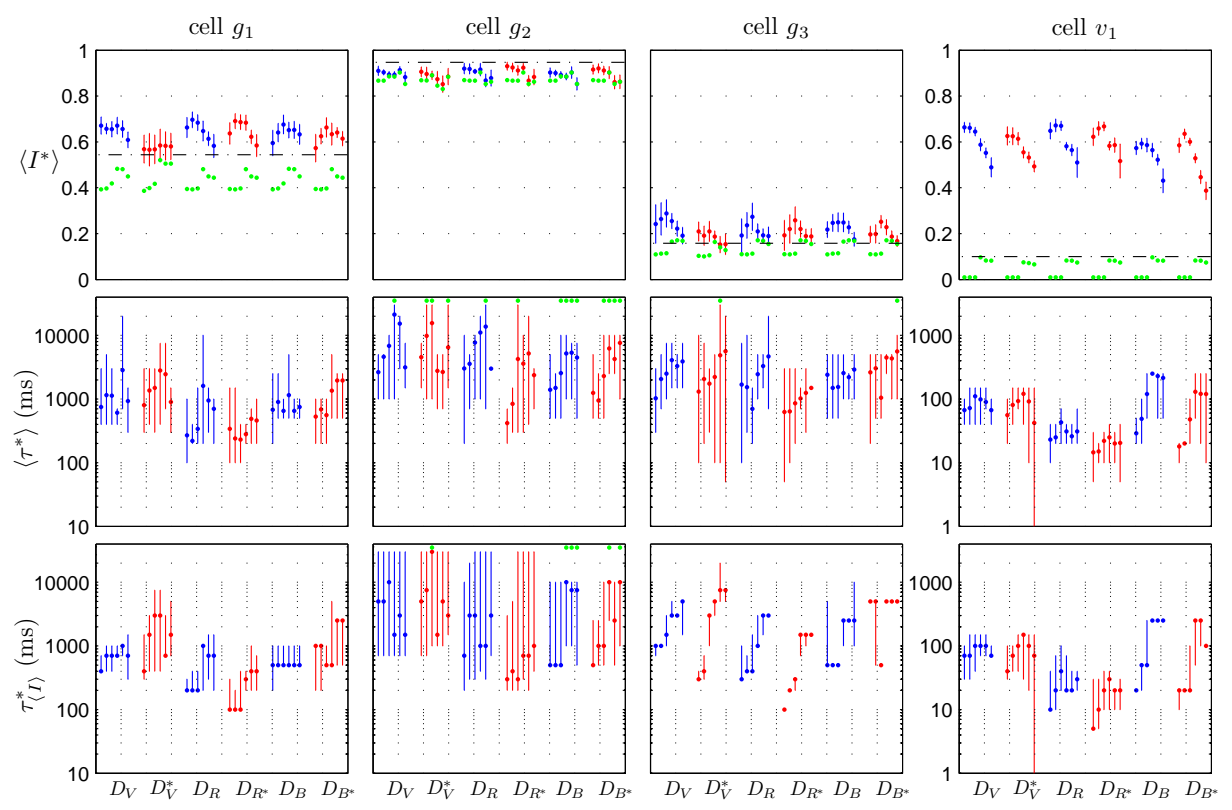

Figure 8: Maximal mutual information $I_{\text {max }}^{*}\left(S^{P}, S\right)$ and optimal time scale $\tau^{*}$ in dependence on the measure and classifier for the exemplary cells. The figure is analogous to Figure 3. For each measure the results are shown for exponents $z=[-8,-4,-2,2,4,8]$, from left to right, with the same color. In the upper row we indicate an estimate of the mutual information obtained without considering temporal coding, $I^{*}(R, S)$ (dotted black line). Details on the calculation of the averages and the error bars are provided in Section 2.1. 


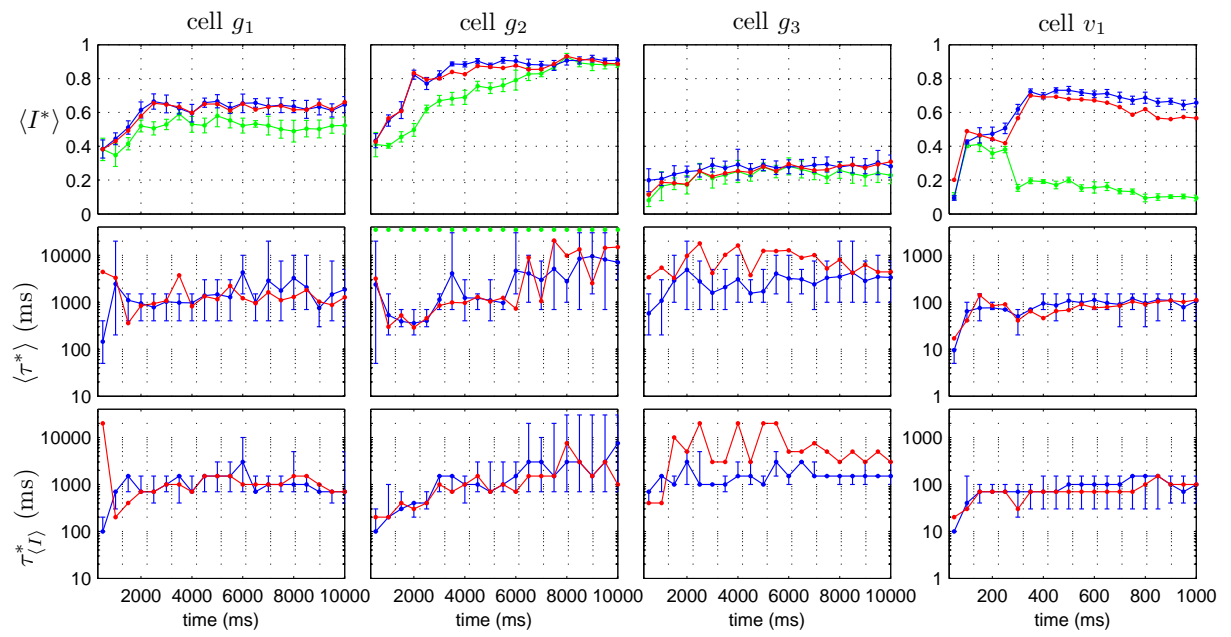

Figure 9: Temporal accumulation of information for the exemplary cells. Dependence of $I_{\max }^{*}\left(S^{P}, S\right)$ and $\tau^{*}$ on the length $L$ of the recordings used in the discrimination analysis. The maximal mutual information $I_{\max }^{*}\left(S^{P}, S\right)$ is shown for $z=-2$ (blue), and for $z=2$ (red). We also show the mutual information obtained by a rate code $I_{\text {count }}^{*}\left(S^{P}, S\right)$ (green). For the optimal time scales $\left\langle\tau^{*}\right\rangle$ and $\tau_{\langle I\rangle}^{*}$, results are display with error bars for $z=-2$ and without for $z=2$. The length $L$ is increased in steps of $500 \mathrm{~ms}$ for the gustatory cells $g_{1}-g_{3}$ and $50 \mathrm{~ms}$ for the visual cell $v_{1}$. Results are shown for $D_{\mathrm{V}} . K=20$ bootstrapping realizations with $M^{\prime}=\frac{3}{4} M$ randomly chosen trials were used. The standard deviations of the mutual information across the $K$ realizations are displayed for $z=-2$ and for $I_{\text {count }}^{*}\left(S^{P}, S\right)$.

pura (1998) found that the discriminability of spatial phases depends on $L$ for V1 visual cells. Since both the gustatory cells and the visual cell respond to transient presentations of constant stimuli, we expect that $L$ plays an important role in the analysis as found for analogous simulations (Section 3.2.2).

In Figure 9 we show the dependence of $I_{\max }^{*}\left(S^{P}, S\right)$ and $\tau^{*}$ on $L$. For all cells the mutual information increases with $L$ until it saturates or slightly decreases again. We compare $I_{\text {max }}^{*}\left(S^{P}, S\right)$ obtained for $z=-2,2$ with $I_{\text {count }}^{*}\left(S^{P}, S\right)$, and observe a different dependence for each cell. For cell $g_{2}$ there is almost no difference among the classifiers, but the difference between $I_{\max }^{*}\left(S^{P}, S\right)$ and $I_{\text {count }}^{*}\left(S^{P}, S\right)$ varies with $L$. For $L=500 \mathrm{~ms}$, the information obtained by rate coding is almost maximal. When $L$ increases, $\Theta$ becomes substantial but it decreases for $L>2 \mathrm{~s}$, leading again to a rate code for $L>8 \mathrm{~s}$. The first $2 \mathrm{~s}$ are almost as informative as the total. For $L=2 \mathrm{~s}, I_{\max }^{*}\left(S^{P}, S\right)>0.8$ is already 
close to the value obtained for $L=10 \mathrm{~s}$. However, $\left\langle\tau^{*}\right\rangle$ and $\tau_{\langle I\rangle}^{*}$ increase with $L$. This resembles example $\mathrm{F}$ of Figure 4 for which information is contained in two different time scales related to the latency code and the rate code. In such cases a single quantity $\tau^{*}$ is not enough to reflect the time scale at which information is contained, and intermediate values of $\tau^{*}$ are obtained when none of the sources of information predominates. For example, for $L=10 \mathrm{~s}$, the information in the first two seconds can still be obtained with a lower $\tau$, however, using the lower $\tau$ would result in contradictory information for the posterior part of the spike trains. This indicates that $\tau^{*}$ depends in a nontrivial way on the distribution of the sources of information across the whole spike trains and the time scale characteristic of these sources.

We also see in Figure 9 that for cells $g_{1}$ and $g_{3} \tau^{*}$ is less dependent on $L$. This suggest that there is no interplay of different time scales. Furthermore, since the values of $I_{\text {count }}^{*}\left(S^{P}, S\right)$ are close to $I_{\max }^{*}\left(S^{P}, S\right)$, the results are similar to the ones shown in Figure 5. For the visual cell $v_{1}$, the pronounced ON and OFF responses are clearly reflected in $I_{\max }^{*}\left(S^{P}, S\right)$. The information increases abruptly with the ON response and then again with the OFF response. By contrast, $I_{\text {count }}^{*}\left(S^{P}, S\right)$ increases with the ON response but goes down with the OFF response, indicating that the total rates are not enough to distinguish the stimuli without resolving the time of the rate increases. After the OFF response $I_{\max }^{*}\left(S^{P}, S\right)$ decays slightly, in particular for the positive exponents. This indicates that only contradictory information is added. Given the dependence of $I_{\text {max }}^{*}\left(S^{P}, S\right)$ and $I_{\text {count }}^{*}\left(S^{P}, S\right)$ on $L$, $\Theta$ is very sensitive to $L$. Before the OFF response a rate code accounts for almost all the information, while afterwards $\Theta$ is high. However, the optimal time scale $\tau^{*}$ increases for $L<150 \mathrm{~ms}$ but then remains constant at a time scale which is neither related to the presentation period nor to the shorter time scale corresponding to the $\mathrm{ON}$ and OFF responses.

\subsubsection{Distribution and redundancy of the information along the spike trains}

Given the above results, it is clear that information is not distributed uniformly along the duration of the recordings. Furthermore, the rate of saturation of the accumulation of information and the level at which it saturates do not only depend on the temporal distribution of the information but also on how redundant it is across different intervals. To complement the study of the dependence of $I_{\max }^{*}\left(S^{P}, S\right)$ and $\tau^{*}$ on $L$, we here examine these quantities for segments of different length and location. We restrict ourselves to cells $g_{2}$ and $v_{1}$, but the conclusions are common to all cells. We consider all nonoverlapping segments with $100,80,40,20,10$, and 5 percentage of the total length of the recordings, thus including the 10 and $2 \mathrm{~s}$ originally studied for the gustatory cells (Di Lorenzo and Victor, 2003). In Figures 10 and 11 we show the shape of $I^{*}\left(S^{P}, S ; \tau\right)$ for classifiers with $z=-2,2$. We 

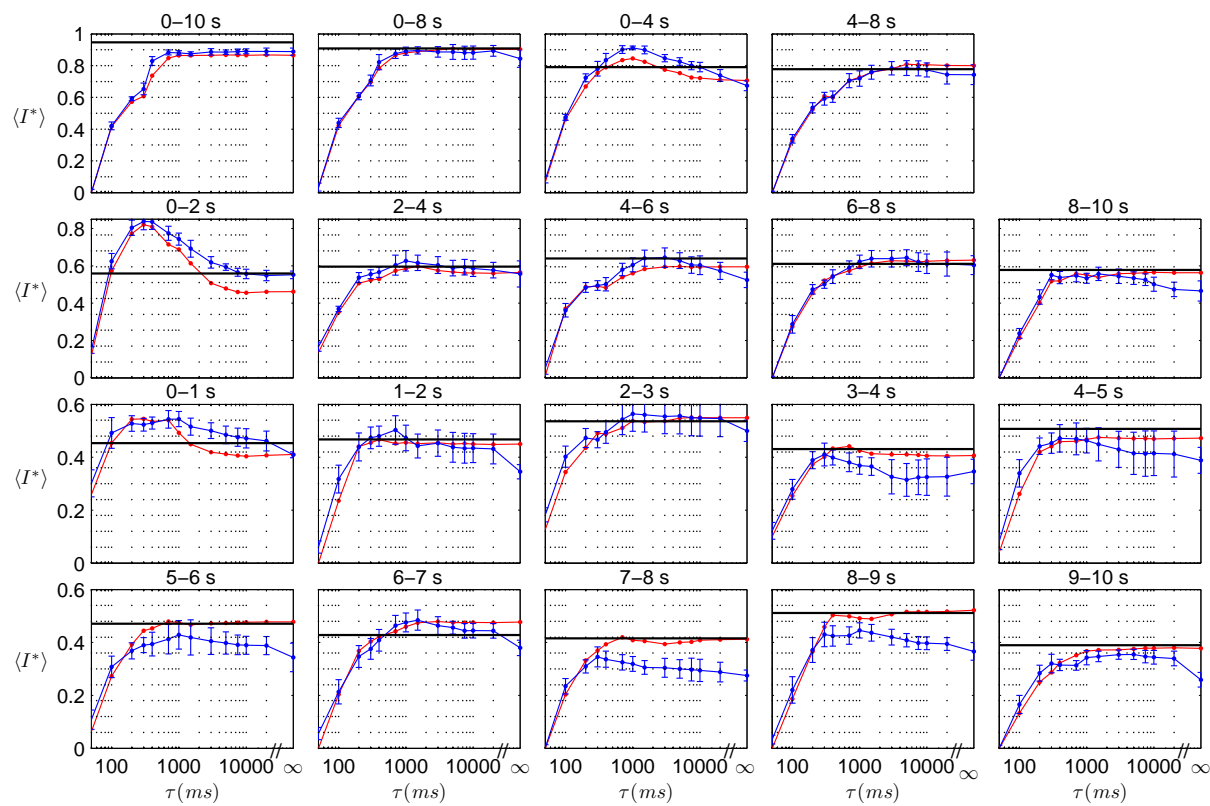

Figure 10: Temporal distribution of information along the recordings for cell $g_{2}$. We show $I^{*}\left(S^{P}, S ; \tau\right)$ for nonoverlapping segments with different location and different length $L$. Row 1: from left to right, segments of $10 \mathrm{~s}, 8 \mathrm{~s}$, and $4 \mathrm{~s}$. Row 2: segments of $2 \mathrm{~s}$. Row 3 and 4: segments of $1 \mathrm{~s}$. We show the results for $z=-2$ (blue), and $z=2$ (red). We also provide an estimate $I^{*}(R, S)$ (black). Results are shown for $D_{\mathrm{V}} . K=20$ bootstrapping realizations with $M^{\prime}=\frac{3}{4} M$ randomly chosen trials were used. The standard deviations of the mutual information across the $K$ realizations are displayed for $z=-2$.

also provide an estimate of $I^{*}(R, S)$, considering the total rate for each segment length, which serves as a reference of the total amount of information obtained without temporal coding.

For cell $g_{2}$, we see that all the $1 \mathrm{~s}$ segments contain similar amounts of information. However, the shape of $I^{*}\left(S^{P}, S ; \tau\right)$ depends on the segment and the classifier. For some segments the same $I_{\max }^{*}\left(S^{P}, S\right)$ is obtained with both classifiers but they produce different shapes of $I^{*}\left(S^{P}, S ; \tau\right)$, and different $\tau^{*}$ and $\Theta$. These differences may be caused in part by the higher estimation noise reflected in the higher variability of $I^{*}\left(S^{P}, S ; \tau\right)$ for short segments of $1 \mathrm{~s}$ as compared to progressively longer segments. Nonetheless, some consistent patterns are observed. For the first second, both classifiers indicate some degree of temporal coding, while for the rest the negative exponent leads to a higher index of temporal 

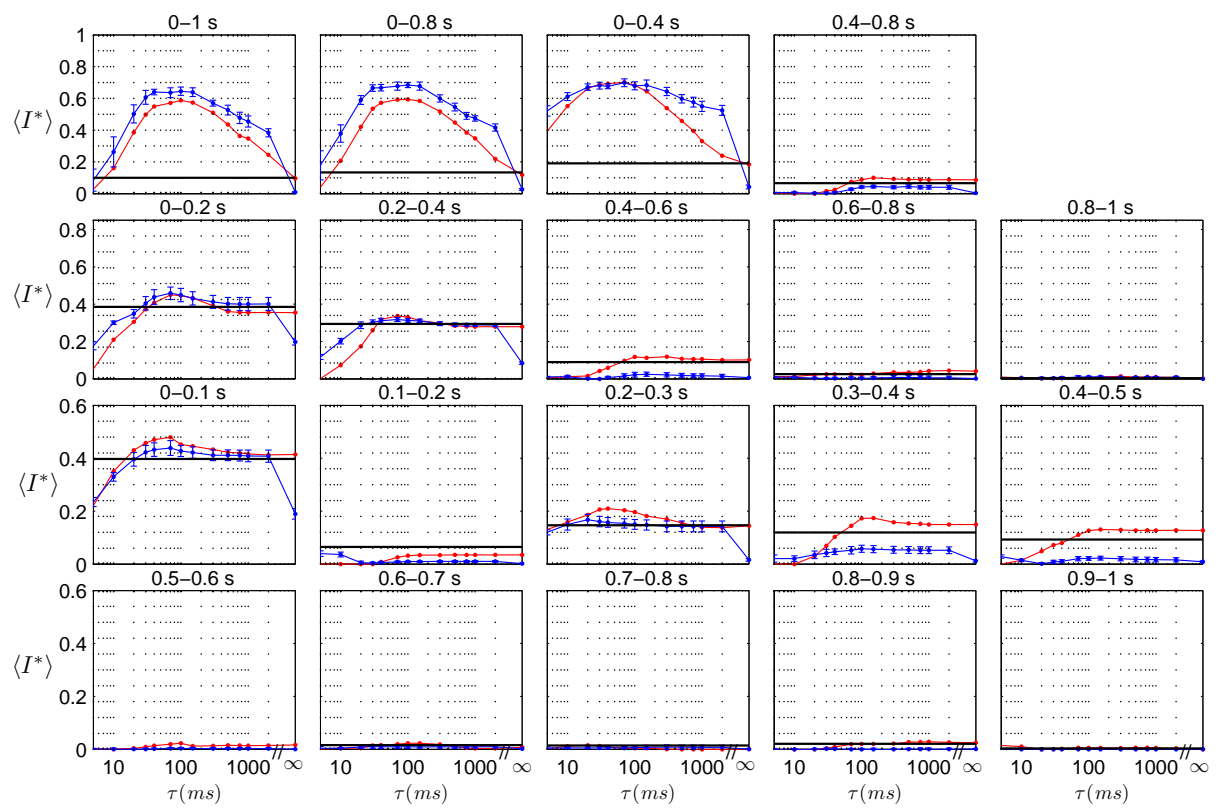

Figure 11: Temporal distribution of information for cell $v_{1}$. Analogous to Figure 10 but notice that the overall length of the spike trains is now $1 \mathrm{~s}$.

coding $\Theta$. In particular, after the phasic response elicited in the first $2 \mathrm{~s}$, for various $1 \mathrm{~s}$ segments the positive exponent lead to higher $I^{*}\left(S^{P}, S ; \tau\right)$ for high $\tau$, nearly matching the estimated $I^{*}(R, S)$. This dependence on the exponent is even higher for $I_{\text {count }}^{*}\left(S^{P}, S\right)$. We found that positive exponents are preferable for the posterior part of the response for all the cells. When examining the segments of length $2 \mathrm{~s}$, the first segment is clearly different from the rest, with temporal coding both indicated by the peak at low $\tau$ and the values of $I_{\max }^{*}\left(S^{P}, S\right)$ higher than $I^{*}(R, S)$. Notice that for the posterior segments the shapes for the two exponents are almost overlapping, in contrast to their divergence for the corresponding segments of 1 s. Furthermore in these segments $I_{\max }^{*}\left(S^{P}, S\right)$ matches $I^{*}(R, S)$. The transition from temporal to rate code is already visible for the first segment of $4 \mathrm{~s}$ where the peak of $I_{\max }^{*}\left(S^{P}, S\right)$ is closer to $I^{*}(R, S)$, and this trend is consolidated for the 8 $\mathrm{s}$ and $10 \mathrm{~s}$ segments.

For cell $v_{1}$ the temporal distribution of information is completely different. Regarding the segments of length $100 \mathrm{~ms}$, the last five segments do not contain any information at all. These are the segments posterior to the OFF response. For the first five segments, the first segment contains the highest amount of information, the second the lowest, and the other three a similar amount. Notice that the seg- 
mentation has not been done taking into account where exactly the ON and OFF responses occur, which means that taking segments located at these specific times could show that information is higher and more concentrated. However, for our purpose, it suffices to show how information arises nonuniformly. For the first, third, and, to a lower degree, the fourth segment, temporal coding retrieves more information. Analogously to cell $g_{2}$, for segments four and five the positive exponent substantially increases the information. Note that for this cell the variability of $I^{*}\left(S^{P}, S ; \tau\right)$ across bootstrapping realizations is lower, given that, although the same percentage of trials are left out, the absolute number of trials is bigger than for $g_{2}$.

The concentration of information during the presentation period is confirmed when examining the segments of $200 \mathrm{~ms}$. For this length $I_{\max }^{*}\left(S^{P}, S\right)$ is only slightly higher than $I^{*}(R, S)$ for the first two segments. By contrast, when these two segments are merged in the first segment of $400 \mathrm{~ms}$, the rate code discriminates clearly worse than if temporal coding is used. The first segment of $400 \mathrm{~ms}$ contains slightly more information than the total of $1 \mathrm{~s}$, in agreement with the decay of the accumulated information observed in Figure 9. For $800 \mathrm{~ms}$ and $1 \mathrm{~s}$ the negative exponent retrieves more information, oppositely to what occurs for the shortest segments.

Apart from revealing the distribution of information along the spike train length, the results of Figures 10 and 11 also implicitly suggest to which degree the information contained in different segments is redundant. To better appreciate how the local information accumulates, we compare the shape of $I^{*}\left(S^{P}, S ; \tau\right)$ for a given segment to the shape of the two subsegments of half length. In Figures 12 and 13 we furthermore indicate for each case the redundancy index (Reich et al., 2001a):

$$
R I=\frac{I_{\max , 1}^{*}+I_{\max , 2}^{*}-I_{\max , j o i n t}^{*}}{I_{\max , 1}^{*}+I_{\max , 2}^{*}-\max \left(I_{\max , 1}^{*}, I_{\max , 2}^{*}\right)} .
$$

If the information of the two subsegments is independent, it will be added so that $I_{\max , j o i n t}^{*}=I_{\max , 1}^{*}+I_{\max , 1}^{*}$, and $R I=0$ in this case. If the information in the two segments is completely redundant there is no new information so that $I_{\text {max } \text {,joint }}^{*}=$ $\max \left(I_{\max , 1}^{*}, I_{\max , 2}^{*}\right)$ and $R I=1$. When $I_{\max , j o i n t}^{*}<\max \left(I_{\max , 1}^{*}, I_{\max , 2}^{*}\right)$ this means there is contradictory information and $R I>1$. Oppositely, for synergistic information $I_{\max , \text { joint }}^{*}>I_{\max , 1}^{*}+I_{\max , 1}^{*}$ and $R I<0$. However, since the denominator $I_{\max , 1}^{*}+I_{\max , 2}^{*}-\max \left(I_{\max , 1}^{*}, I_{\max , 2}^{*}\right)=\min \left(I_{\max , 1}^{*}, I_{\max , 2}^{*}\right)$, this index is especially sensitive when low information is found in one of the half segments. Here we use it to qualitatively indicate the accumulation of information.

In Figure 12, for cell $g_{2}$, the average $R I$ is about 0.6 or higher for the segments of length $1 \mathrm{~s}$, indicating that there is a substantial redundancy between the information. For the first second, which shows a clear peak indicating temporal coding, the 

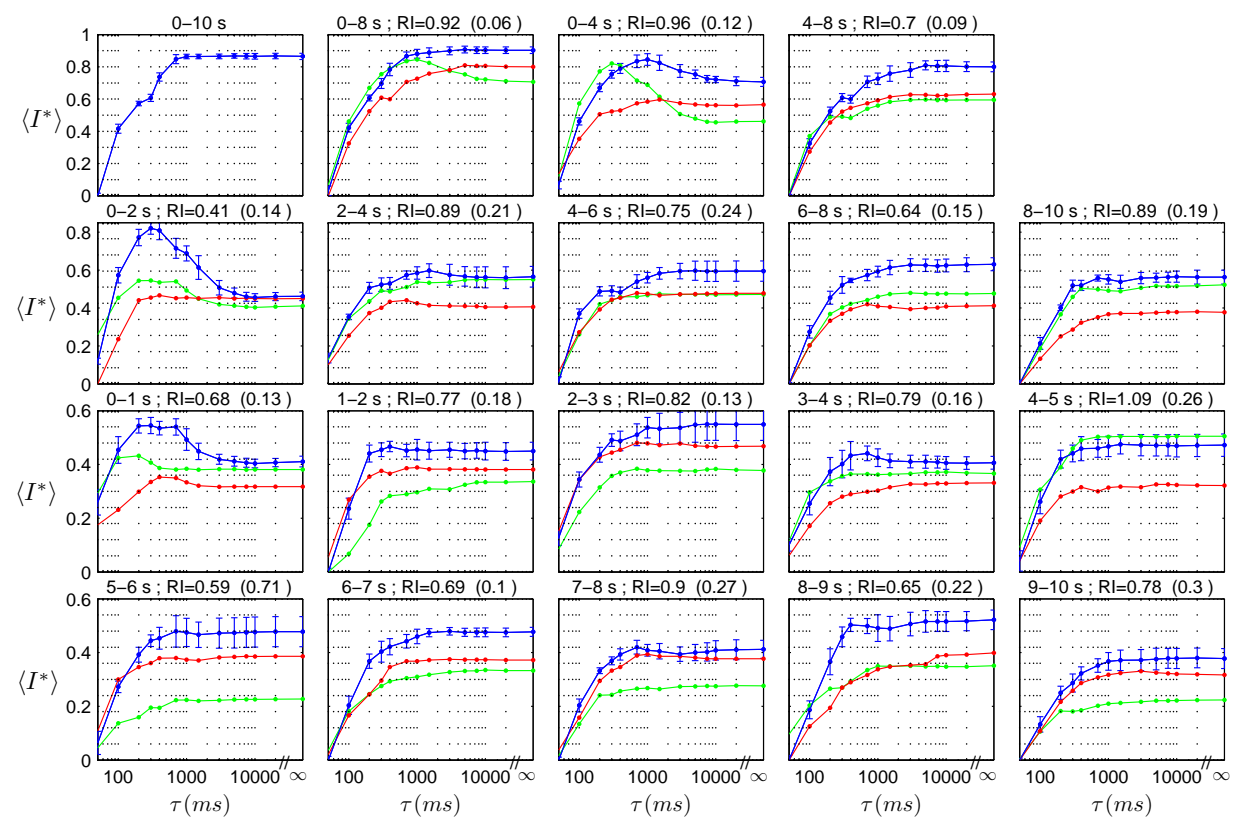

Figure 12: Redundancy of information along the recordings for cell $g_{2}$. $I^{*}\left(S^{P}, S ; \tau\right)$ for nonoverlapping segments with different location and different length $L$. The structure of the figure is analogous to Figure 10. However, apart from the mutual information $I^{*}\left(S^{P}, S ; \tau\right)$ for each segment (blue), also the mutual information for the two nonoverlapping subsegments of half length are shown, the first in green, the second in red. Moreover, we provide for each segment the redundancy index (Equation 10) quantifying the redundancy between the two subsegments and in parentheses its standard deviation across bootstrapping realizations. Results are shown for $D_{\mathrm{V}}$ and $z=2 . K=20$ bootstrapping realizations with $M^{\prime}=\frac{3}{4} M$ randomly chosen trials were used. The standard deviations of the mutual information across the $K$ realizations are displayed for the joint segments. 

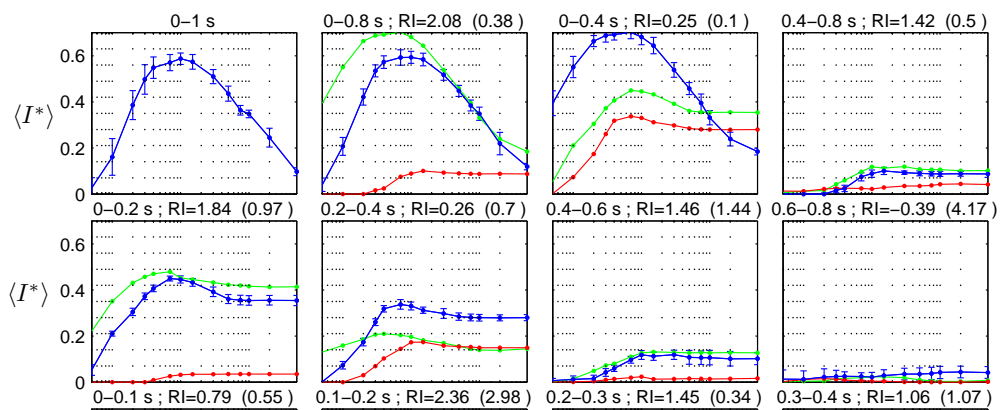

$0.4-0.6 \mathrm{~s} ; \mathrm{Rl}=1.46(1.44) \quad 0.6-0.8 \mathrm{~s} ; \mathrm{Rl}=-0.39(4.17) \quad 0.8-1 \mathrm{~s} ; \mathrm{Rl}=2.05(1.07)$

$\left\langle I^{*}\right\rangle$
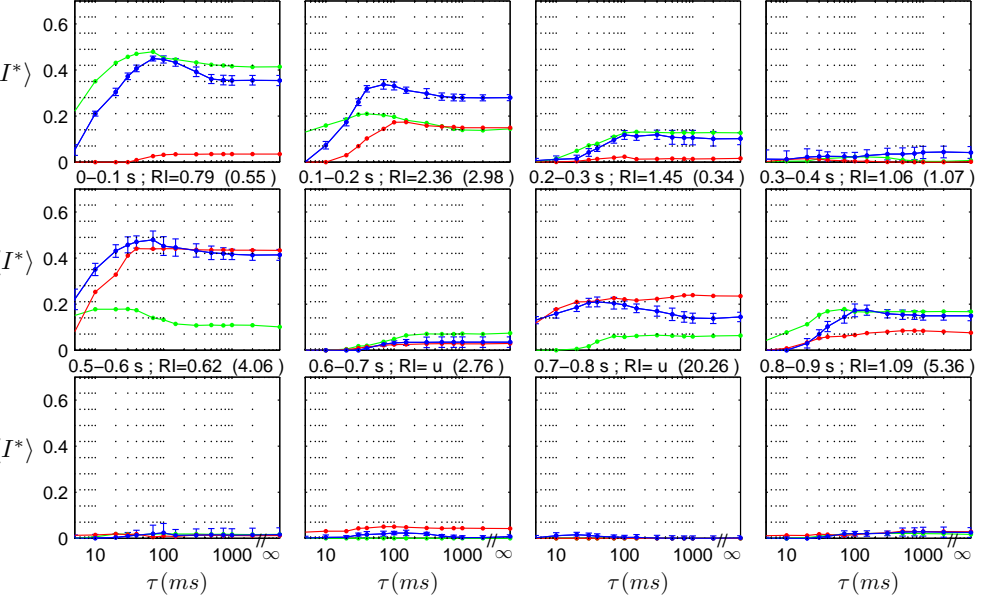

$0.4-0.5 \mathrm{~s} ; \mathrm{Rl}=0.17(0.42)$
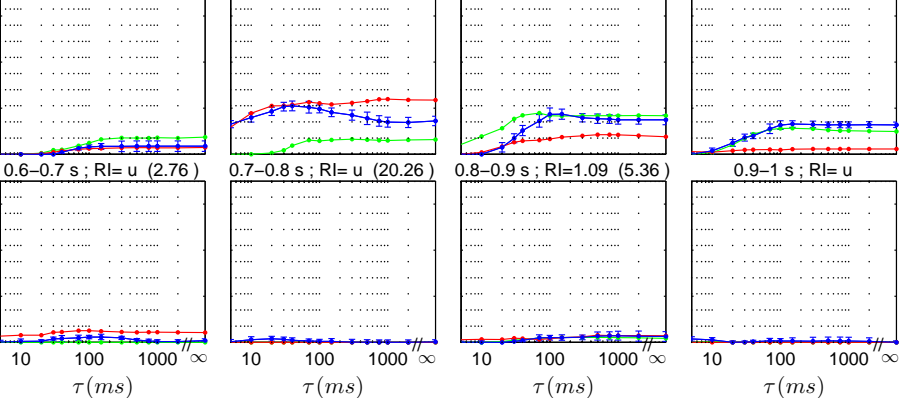

Figure 13: Redundancy of information along the recordings for cell $v_{1}$. Analogous to Figure 12 but notice that the overall length of the spike trains is now $1 \mathrm{~s}$. We indicate with $R I=u$ that the redundancy index is undefined. 
two subsegments are more consistent with a rate code. The standard deviation of $R I$ across the bootstrapping realizations is about 0.2 for these segments, although for the segments $5-6 \mathrm{~s}$ and $9-10$ it is higher, coinciding with the obtention of the lowest mutual information values for one of the subsegments. For the first $2 \mathrm{~s}$, the lowest redundancy index $(R I=0.41)$ over all time scales is obtained, indicating the higher independence of the information extracted from the subsegments. By contrast, for the first $4 \mathrm{~s}$ information is almost completely redundant $(R I=0.96)$. The same occurs for the segment of $8 \mathrm{~s}$. This is consistent with Figure 9 concerning the saturation of the accumulation of information. The phasic response contains almost all the nonredundant information in the first $2 \mathrm{~s}$. Nonetheless, this information can also be retrieved from the posterior response where it is distributed more uniformly and in a less redundant way.

For cell $v_{1}$, we see in Figure 13 that $R I$ is much more variable and we find negative values and values above 1 . For three segments of length $100 \mathrm{~ms} R I$ is not well defined because the information is zero in one of the subsegments. We see that almost all the information is contained in the segments $50-100 \mathrm{~ms}, 250$ $300 \mathrm{~ms}$ and 300-350 ms. The first interval covers the ON response, and the other two cover the OFF response. These segments contained information which is quite independent, as reflected in the low average $R I$ values for the second segment of $200 \mathrm{~ms}(R I=26)$ and the first segment of $400 \mathrm{~ms}(R I=0.25)$. The part of the spike trains after the OFF response provides only contradictory information as evidenced by the $R I$ of the $800 \mathrm{~ms}$ segment. Given that for cell $v_{1}$ low values of the mutual information are involved in the majority of the $R I$ calculations, we should not interpret negative or values above 1 as indicating the existence of synergistic or contradictory information, respectively, but as an effect of the sampling noise. This is also suggested by the high variability across bootstrapping realizations. Nonetheless, the variability is particularly low for the segment $0-0-4 \mathrm{~s}$, where the independence of the information from the $\mathrm{ON}$ and OFF response is quantified.

Overall, for cell $g_{2}$ the information is almost homogeneously distributed. For cell $v_{1}$ it is concentrated at the times of the onset and offset of the stimulus presentation. The analysis confirms the observed accumulation of information, indicating that the first $L \sim 2 \mathrm{~s}$ for the gustatory cells and the first $L \sim 400 \mathrm{~ms}$ for the visual cell already contain almost all the nonredundant information. Nonetheless, the information contained in the posterior part of the responses varies from cell to cell. For cell $g_{2}$, almost the same information could be obtained excluding the first $2 \mathrm{~s}$. By contrast, for cell $v_{1}$, almost no information is contained after the first $400 \mathrm{~ms}$. 


\section{Discussion}

In this article we dealt with the use of time scale parametric spike train distances to study the information and discriminative precision of neural responses. For this purpose, we studied several measures commonly used to quantify the dissimilarity between spike trains. We started by comparing these measures using time-independent Poisson spike trains to characterize the dependence on the time scale parameter $\tau$ (Section 3.1). This characterization is necessary to understand the dependence of the mutual information between the stimuli and the predicted stimuli $I^{*}\left(S^{P}, S ; \tau\right)$ (Equation 4) on the time scale parameter. We then evaluated the influence of different factors in the discrimination analysis. In particular, we considered the dependence of the mutual information and the discriminative precision on the measure and the selected classifier, as well as on the length of the spike trains used for the discrimination analysis.

We showed that the mutual information $I_{\text {max }}^{*}\left(S^{P}, S\right)$, obtained from the maximization across the time scales, is more sensitive to the classifier than to the measure. This result was consistent for simulated time-dependent Poisson spike trains (Section 3.2.1) and for exemplary experimental data comprising recordings from gustatory and visual neurons (Section 3.3.1). In particular, a simple distance based on binning led to similar results as the more elaborated spike train distances. This suggests that the preeminent ingredient of the discrimination analysis is the use of a classifier to avoid the estimation of the joint probability distribution of stimuli and responses $p(\mathbf{R}, S)$ (Equation 1). This is in contrast to the emphasis usually put on the spike train distances. In particular, the use of a metric specific for point processes like the Victor distance (Victor and Purpura, 1996) or a metric in an Euclidean space like for the binning distances is not so determinant for the discrimination performance as the classifier, given that in this case the binning procedure is not used to construct a high-dimensional space for the probability distributions (Strong et al., 1998). Notice that here we restricted ourselves to the type of classifier originally proposed by Victor and Purpura (1996), which has been also the most used in studies applying the discrimination analysis. The possibility to use more elaborated classifiers in the discrimination analysis (e. g. Schneider and Woolley, 2010) do not invalidate our conclusion. On the contrary, our results suggest that one should maximize the mutual information not only across the time scales but also across classifiers.

Regarding the discriminative precision, we observed that the time scale $\tau^{*}$ for which the mutual information is maximal results from a nontrivial interplay of the different sources of temporal structure, their redundancy, as well as from the different time scales at which the information is optimally extracted in each source. For example, we showed that for simulated time-dependent Poisson spike trains, 
$\tau^{*}$ does not depend directly on the time scale of the modulation in the rate. It is determined by several factors including the local and global balance between the rates in response to each stimulus. Furthermore, $\tau^{*}$ and the index of temporal coding $\Theta$ (Equation 6) are more sensitive to the measure and classifier than the mutual information itself.

This study is more related to Victor and Purpura (1997) than to subsequent characterizations of the time scale parametric spike train distances (e. g. Schrauwen and van Campenhout, 2007; Paiva et al., 2010) since we investigate how informative are the results of the discrimination analysis about the neural code. For example, Schrauwen and van Campenhout (2007) and Paiva et al. (2010) studied with simulated Poisson processes the dependence of the discrimination performance on the use of different kernels for convoluting the spike trains. They did not carry out a fine sampling of the time scale parameter nor addressed the interpretation of the optimal time scale in terms of characteristic time scales of the code or the stimuli. Schrauwen and van Campenhout (2007) used both $k$-nearest neighbors and support vector machines as classifiers. Consistently with our results they found that the discrimination performance was more dependent on the classifier than on the spike train distance or kernel selected. Paiva et al. (2010) also showed that the discrimination performance has a minor dependence on the convolution kernels. They found that a measure of correlation based on binning had a worse performance than the analogous but binless measure $D_{\mathrm{S}}$. However they evaluated the discrimination performance in a classifier-free manner focusing on the influence of the spike train distances. In particular, they used an index of the normalized difference between average intra and inter-cluster distances. As we commented when examining the dependence of the spike train distances on the time-independent Poisson rates in Section 3.1, the magnitude of this difference may be not conclusive with regard to the classification performance.

Our work contributes to the methodological study of the discrimination analysis carried out in Victor and Purpura (1997) in several ways. We here more explicitly show the impact of the classifier which was already suggested in the exemplary application of the discrimination analysis to Gamma processes with different rates. Besides that we compare the discrimination analysis for different time scale parametric spike train distances all based on spike train synchrony. This is in contrast to the comparison of the Victor distances based on spike and intervals similarity respectively, which assume a different nature of the code. Furthermore, we also characterize the influence of other factors comprising the spike train length used in the discrimination analysis, the accumulation of information and the redundancy of the sources of information. These different aspects had been examined in some experimental studies (e. g. Machens et al., 2001; Di Lorenzo and Victor, 2003; Reich et al., 2001b) but, to our knowledge, had never been considered in a methodolog- 
ical study to discuss the interpretation of the results of the discrimination analysis in terms of the properties of the neural code.

\subsection{The dependence on the classifier and the measure}

To our knowledge, the dependence of the mutual information on the classifier, determined by the exponent $z$ in Equation 2, has not been addressed in detail before. Victor and Purpura (1997) showed that, for simple Gamma processes, the shape of $I\left(S^{P}, S ; \tau\right)$ changes with $z$. This affects the determination of the optimal time scale $\tau^{*}$ as well as the significance of temporal coding quantified by $\Theta$. However, the particular exponent used is usually not specified in experimental studies (Victor and Purpura, 1998; Machens et al., 2001; Aronov et al., 2003; Di Lorenzo and Victor, 2003; Samonds and Bonds, 2004; Di Lorenzo and Victor, 2007; Roussin et al., 2008; Huetz et al., 2009). When explicitly stated (Mechler et al., 1998; Macleod et al., 1998; Huetz et al., 2006), it is usually not motivated. Victor and Purpura (1996), presented the results for $z=-2$ and mention that similar results were obtained for $z=-8, \ldots-1$, and Reich et al. (2001b) provided an analysis of the robustness of the median distance for Equation 2 when few spikes are present in each spike train. We showed (Section 3.2.2) that $I^{*}\left(S^{P}, S ; \tau\right)$ should be maximized not only across time scales $\tau$, but across different classifiers, for example for different values of $z$. Furthermore, for the comparison of $I_{\max }^{*}\left(S^{P}, S\right)$ and $I_{\text {count }}^{*}\left(S^{P}, S\right)$, both should be maximized separately.

Alternatively, to avoid the parametric dependence on the exponent another classifier has been commonly used (e. g. Machens et al., 2003; Narayan et al., 2006). For each stimulus $S_{i}$ the spike train of one of the trials, $s_{i, j_{T}}$, is chosen as a template and each spike train $s_{i^{\prime}, j^{\prime}}$ is assigned to the stimulus with the nearest template. This procedure is repeated for $K_{T}$ randomly chosen templates. The discrimination performance is quantified by the percentage of correct classifications. Notice that the maximal mutual information and the percentage of correct classifications are not biunivocally related (Thomson and Kristan, 2005) and quantify different aspects of the representation of the stimuli in the responses. Machens et al. (2003) indicated that the use of different supervised and unsupervised classifiers did not affect the results. However, other studies using the percentage of correct classifications to evaluate the discrimination do not report any comparison with other classifiers (Grewe et al., 2003; Narayan et al., 2006; Wohlgemuth and Ronacher, 2007; Wang et al., 2007; Billimoria et al., 2008). Although avoiding the selection of $z$, the optimality of the classifier based on the random selection of templates may differ for different time scales, cells, or conditions.

It is also common practice to apply the discrimination analysis for only one particular measure in application to experimental data (e. g. Huetz et al., 2006; 
Roussin et al., 2008). In other cases various measures are used to verify if the results are independent of the measure (Machens et al., 2003; Narayan et al., 2006), or to compare their performance (Wang et al., 2007). In particular, the two different families of spike train distances based on spikes and intervals (Victor and Purpura, 1996), are usually considered together to examine the nature of the code (e. g. Victor and Purpura, 1996; Di Lorenzo and Victor, 2003; Samonds and Bonds, 2004).

\subsection{The dependence on the length of the spike trains for different codes}

In Section 3.2.2, we showed that for pure latency coding of the stimuli $I_{\max }^{*}\left(S^{P}, S\right)$ is almost independent of the length of the after-transient interval, and $\tau^{*}$ reflects the time scale of the latency difference. However this direct interpretation does not hold in general. When information is extracted at different time scales, the discriminative precision arises from the particular balance between how well for a particular $\tau$ the different sources of information can be extracted. This has been confirmed by the examination of the accumulation and distribution of information along the spike trains for the experimental recordings in Sections 3.3.2 and 3.3.3. Choosing a particular $L$ implies some assumptions about which portion of the responses is informative, but also determines which is the best $\tau$ to retrieve this information. Different classifiers can be better for different $L$, leading to counterintuitive results like in Figure 5, unless results across several classifiers are scrutinized. This led us to also consider a direct calculation of $I^{*}(\mathbf{R}, S)$, the normalized mutual information between the stimuli and the responses, calculated directly from Equation 1. The calculation is straightforward for simulations for which a large number of data can be generated to avoid estimation biases. Since the mutual information obtained from the classifier $I_{\max }^{*}\left(S^{P}, S\right)$ is a lower bound of $I^{*}(\mathbf{R}, S)$, this helps to evaluate the optimality of the classifiers.

\subsection{The application of discrimination analysis to experimental data: transient constant vs. time-dependent stimuli}

The measures studied here by construction cannot discriminate between temporal structure caused by correlations with the stimuli, patterns associated to temporal encoding as defined by Theunissen and Miller (1995), or the effect of refractory periods or short-term adaptation. However, depending on the experimental setting, some of these sources are expected to be more relevant and should be taken into account to interpret the results. In general, two main scenarios can be distinguished.

In one case, transient constant stimuli are presented and the responses are recorded including the period of the stimulus presentation and potentially some period after the stimulus presentation (e. g. Victor and Purpura, 1996, 1998; Reich et al., 2001b; Aronov et al., 2003; Di Lorenzo and Victor, 2003; Di Lorenzo 
et al., 2009). In Section 3.2.2 we investigated the influence of the length of the spike trains $L$ used in the discrimination analysis for simulations of different types of responses to transient presentation of constant stimuli. In Section 3.3 we analyzed exemplary cells responding to this type of stimuli. For this scenario, since the stimulus is constant, the temporal structure is not determined by correlations with the stimulus apart from those elicited by the onset and the offset. Therefore, we used the term temporal coding in the broad sense of any contribution of time to determine the information in the responses as in Hallock and Di Lorenzo (2006), in contrast to the more restrictive definition of Theunissen and Miller (1995). The temporal structure is mainly caused by the phasic response and short-term adaptation (see Hallock and Di Lorenzo, 2006, for an overview of the different sources of temporal coding for the cells of the gustatory system). The importance of the transient presentation in the temporal coding was demonstrated by Mechler et al. (1998) for the coding of contrast by $V 1$ neurons. After the phasic response the activity depends on the influence of recurrent and feedback circuits (Victor and Purpura, 1998), which contributes to the dependence of the results of the discrimination analysis on $L$.

Furthermore, for the constant stimuli, the property of the stimulus which is assumed to cause the response is implicitly selected in the construction of the set. For example, for the experimental data analyzed in Section 3.3, the taste quality differentiates the stimuli presented to the gustatory cells, while the spatial phase changes for the stimuli presented to the visual cell. Other properties of the stimulus, for example the contrast for the visual stimuli, are kept constant or assumed to have no causal effect in the responses. In this case, the mutual information between the stimuli and the predicted stimuli is a lower bound of the mutual information between the stimuli and the response. Accordingly, the main problem of interpretation when obtaining a low value for the mutual information is that with $I_{\text {max }}^{*}\left(S^{P}, S\right)$ one evaluates at the same time the capability of the neurons to discriminate and the capability of the spike train distances and the classifier to correctly retrieve any available information. Low values can be due to an unappropriate measure or classifier, but also because the cell is insensitive to the selected property of the stimulus which distinguishes the different stimuli in the set. In a considerable part of the studies applying the discrimination analysis, low values of $I_{\text {max }}^{*}\left(S^{P}, S\right)$ have been obtained. For example, an average $I_{\max }^{*}\left(S^{P}, S\right)<0.3$ was extracted in Victor and Purpura (1996), Victor and Purpura (1997), Victor and Purpura (1998), Mechler et al. (1998), Reich et al. (2001b), Samonds and Bonds (2004), and Di Lorenzo and Victor (2007). In other cases average values were higher, for example $I_{\max }^{*}\left(S^{P}, S\right)>0.6$ for Di Lorenzo and Victor (2003), Roussin et al. (2008), and Di Lorenzo et al. (2009), but still low values were found for some cells. 
For the other scenario time-dependent stimuli are used. Discrimination analysis has mainly been applied in this context to discriminate among naturalistic sounds (Machens et al., 2001, 2003; Narayan et al., 2005, 2006; Huetz et al., 2006; Wohlgemuth and Ronacher, 2007; Huetz et al., 2009). In this context, the timedependent stimuli are regarded as a discrete set of different stimuli rather than simply different realizations from an underlying common probability distribution. For example, in studies on the time scale of discrimination of natural sounds in songbirds (e. g. Narayan et al., 2006; Wang et al., 2007), each song presented to a songbird is supposed to be behaviorally relevant and to contain subunits formed by temporal sequences of motifs composed by complex spectrotemporal features that are specific for different singers (Gentner, 2008). Neurons with spectrotemporal receptive fields have been identified in the birds auditory system (Theunissen et al., 2000; Nagel and Doupe, 2008). Accordingly, the temporal structure of the spike train reflects mainly the correlation with the time-dependent stimulus, the particular sequence of spectrotemporal features to which the neuron is sensitive. (Narayan et al., 2005; Billimoria et al., 2008). For neurons with different receptive field the time-dependent stimulus constitutes a different time-dependent profile of relevant features. This results in a highly variable mutual information and discriminative precision across cells (Narayan et al., 2005, 2006; Wang et al., 2007; Schneider and Woolley, 2010).

For time-dependent stimuli there is no clear identification of what is causally influencing the responses. The stimuli forming the set $\{S\}$ are not distinguisable by one property, like the spatial phase, but they are behaviorally relevant complex entities, consisting of a sequence of features resulting in a particular timedependent profile of relevant features for each cell. In this case the mutual information between stimuli and predicted stimuli is not a lower bound of the mutual information between stimuli and responses, and should be considered simply as a measure of discrimination performance, similar to the percentage of correct classifications (Machens et al., 2003; Narayan et al., 2006). The percentage of correct classifications cannot be directly compared to the normalized mutual information. However, qualitatively, higher levels of discrimination performance have been reported when analyzing time-dependent stimuli. This higher levels of performance can be understood given that in the time-dependent stimuli the evidence of the difference between the stimuli increases with time, if the cell is sensitive to some features in the stimuli. For example, percentages higher than $75 \%$ were reported in Machens et al. (2001, 2003); Narayan et al. (2005, 2006). The discrimination level depends on the influence of the distribution of these relevant features specific for each cell across time and across stimuli. This means that the statistics of the stimuli, combined with the properties of receptive field and the tuning curve of the cell have to be considered together with the sensitivity of the similarity measures and 
the appropriateness of the classifier to interpret the discrimination performance. Therefore, an analysis of the influence of the length $L$ of the spike trains in the discrimination accuracy depends crucially on the specific time-dependent profile resulting for each song and cell. To restrict the number of influencing factors on the estimation of the mutual information and the discriminative precision, we focused on examples reproducing the responses to transient constant stimuli.

\subsubsection{Discrimination analysis for transient constant stimuli}

In Section 3.3 we studied exemplary spike trains elicited by transient constant stimuli. In Section 3.3.1 we showed that the dependence on the measure and classifier used was consistent with the results for the simulated data discussed in Section 3.2.1. We again observed that it is harder to estimate and evaluate the discriminative precision and significance of the temporal coding than the mutual information. We furthermore included in our analysis a direct estimate of $I^{*}(R, S)$, the mutual information between the stimuli and the total rates in the spike trains. With the number of trials available it was not possible to deal with a space $\mathbf{R}$ of high dimensionality, derived from a finer binning of the spikes. However, calculating $I^{*}(R, S)$ helps to verify the relevance of the temporal coding. Since $I_{\text {count }}^{*}\left(S^{P}, S\right)$ is only a lower bound of $I^{*}(R, S)$, one should check if $I^{*}(R, S)$ is higher than $I_{\text {max }}^{*}\left(S^{P}, S\right)$. To our knowledge, such a control including $I^{*}(R, S)$ in the analysis has only been carried out by Huetz et al. (2006).

We studied the influence of the length $L$ of the spike trains used for the discrimination analysis. We found some similarities between the simple examples of Section 3.2.2 and the experimental data. In particular, for cell $g_{2}$, we showed the effect of the existence of information at different time scales, as in example B of Section 3.2.2, where both the latency and the total spike counts were informative. We argued that one quantity, $\tau^{*}$, is not enough to reflect the time scales at which information is contained in the code. Its value depends in a nontrivial way on the balance between how the sources of information are distributed in different locations of the spike trains and on the time scale characteristic of these sources.

The dependence of the discrimination analysis on the length of the responses has been studied before (Machens et al., 2001, 2003; Narayan et al., 2005, 2006; Wang et al., 2007; Wohlgemuth and Ronacher, 2007). However, our analysis differed in several ways. First, in all these studies time-dependent stimuli were used, so that increasing $L$ also increases the amount of evidence in the stimuli contributing to their discrimination. Therefore in these cases how the discrimination improves depends more on the statistics of the different time-dependent profiles than on the encoding process. Second, in these studies the dependence on $L$ is shown for a fixed value of $\tau$, usually corresponding to $\tau^{*}$ extracted for a particular sufficiently large length of the spike trains (for which the mutual information is high). Oppo- 
sitely, in Figure 9, the values of $I_{\max }^{*}\left(S^{P}, S\right)$ were maximized independently for each value of $L$. As indicated by the variability of $\tau^{*}$ on $L$, using a fixed $\tau$ can lead to an underestimation of the available information. Narayan et al. (2006) derived an estimate of the time scale of temporal integration fitting the cumulative curves to an exponential. They discussed the existence of two distinct time scales in the discrimination, one related to the discriminative precision and the other to the time of integration. A longer integration time scale can result from the underestimation of $I_{\text {max }}^{*}\left(S^{P}, S\right)$ when $\tau$ is fixed for all $L$.

To better understand the accumulation of information, we examined which amount of information can be extracted locally in different parts of the spike trains and their redundancy (Section 3.3.3 ). We saw that in different segments the best classifier differs. In some cases, even if $I_{\max }^{*}\left(S^{P}, S\right)$ was almost equal, different classifiers indicated different discriminative precision and importance of the temporal coding. It was not possible to relate the discriminative precision to the way information was temporally distributed.

For transient constant visual stimuli, Reich et al. (2001b) already carried out a discrimination analysis considering separately different parts of the responses. They compared the information extracted in the transient part of the response, on the tonic part, and the off part of the response -after the stimulus has been removed. Although they did not focus on the redundancy of the information in these distinct parts, they already indicated that the information contained in the time of the first spike and all the other sources of information were redundant, so that the first spike was even more informative than the full response for some cells. In another study, Nelken et al. (2005) used other estimators of the mutual information to identify a minimal ensemble of properties of the responses that contains all the available information. It was shown that the total rates and the mean of the spike times contained together the same information as the full responses. This shows that examining which parts of the responses contribute the most to the mutual information provides more information about the time scales and the nature of the encoding than the discriminative precision. The way information is distributed and combined in different parts of the responses and in different time scales can be too complex to be faithfully reflected by just one quantity.

\subsection{Spike train distances to study population coding}

Extensions for population codes of the Victor distance (Aronov et al., 2003) and of the van Rossum distance (Houghton and Sen, 2008) have been proposed, and our conclusions derived for single neurons are applicable to these measures as well. These extensions have been developed adding a new parameter that quantifies if the interactions between cells in the population contribute to a better discrimination of the stimuli. In one extreme of the parameter range, the spike train distances are 
insensitive to which neuron fires each spike, while in the other the distances are calculated separately for each cell. To determine the contribution of interactions to discrimination one has to take as a reference the maximal discrimination achievable without considering the interactions. Since $I_{\max }^{*}\left(S^{P}, S\right)$ is only a lower bound of $I^{*}(\mathbf{R}, S)$, this reference depends on the measure and classifier used. Accordingly, our results also point to the difficulty to interpret the parameter associated with population coding.

\section{Conclusions}

We analyzed the factors influencing a discrimination analysis that combines a measure of spike train similarity with a classifier. This analysis provides the mutual information between stimuli and predicted stimuli, and an optimal time scale of discrimination. We showed that the mutual information obtained from the classification is more robust than the discriminative precision, or any measure quantifying the relevance of temporal coding, to the factors and parameters influencing the discrimination analysis. This mutual information is a lower bound of the mutual information between the stimuli and the responses when each category of the classifier is associated with a constant stimuli. By contrast, it is generally difficult to interpret the meaning of the discriminative precision related to the optimal time scale which, strictly, indicates nothing more than the parameter for which the maximal mutual information is obtained.

Spike train distances are generally useful to quantify the reliability of the neural responses of a single cell (e. g. Kreiman et al., 2000), or to evaluate the performance of single neuron models to reproduce the spike times elicited by a stimulus (Jolivet et al., 2008). Furthermore, they have been often applied to characterize the temporal structure in the spike trains relevant for the neural code (e. g. Victor, 2005, and references therein). However, we showed that it is difficult to interpret the results obtained using time scale parametric spike train distances to decipher the neural code or to identify biologically meaningful time scales. According to our results, the time scale parametric nature of these measures is mainly an advantage in the sense that it allows maximizing the mutual information across a whole set of measures with different sensitivities. This is in contrast to the view that the main advantage is the possibility to calculate the discriminative precision and examine temporal coding. Therefore, to find the maximal mutual information, more elaborated classifiers could be employed. Similarly, for spike trains with a rate profile containing more than one relevant time scale, like in the presence of bursting, time scale adaptive and parameter free spike train distances (Kreuz et al., 2007, 2009, 2011) could be more appropriate to obtain the maximal mutual information. 


\section{Acknowledgments}

DC is supported by the grant 2010FI-B2 00079 of the "Comissionat per a Universitats i Recerca del Departament d'Innovació, Universitats i Empresa de la Generalitat de Catalunya i del Fons Social Europeu" and grant 2008BE1 00166 of the "Comissionat per a Universitats i Recerca del Departament d'Innovació, Universitats i Empresa de la Generalitat de Catalunya". TK has been supported by the Marie Curie Individual Outgoing Fellowship STDP, project No 040576 and acknowledges support by the Italian Ministry of Foreign Affairs regarding the activity of the Joint Italian-Israeli Laboratory on Neuroscience. RGA acknowledges grant BFU2007-61710 of the Spanish Ministry of Education and Science. DC thanks Henry D. I. Abarbanel for his stay at the Institute for Nonlinear Science, UCSD. DC thanks Larissa Albantakis for helpful comments on the manuscript. DC and TK thank Timothy Q. Gentner, Emily Caporello and Jason V. Thompson for fruitful discussions. We thank the Laboratory of Neuroinformatics headed by Dr. Daniel Gardner at Weill Medical College of Cornell University for the data delivered via neurodatabase.org, a neuroinformatics resource funded by the Human Brain Project (http://neurodatabase.org).

\section{References}

Aronov D, Reich DS, Mechler F, Victor JD. Neural coding of spatial phase in v1 of the macaque monkey. J Neurophysiol 2003;89:3304-27.

Bialek W, Rieke F, de Ruyter van Steveninck R, Warland D. Reading a neural code. Science 1991;252(5014):1854-57.

Billimoria CP, Kraus BJ, Narayan R, Maddox RK, Sen K. Invariance and sensitivity to intensity in neural discrimination of natural sounds. J Neurosci 2008;28(25):6304-08.

Borst A, Theunissen FE. Information theory and neural coding. Nature Neurosci 1999;2(11):947-57.

Cover TM, Thomas JA. Elements of Information Theory. John Wiley and Sons, 2nd edition; 2006.

Dauwels J, Vialatte F, Weber T, Cichocki A. Quantifying statistical interdependence by message passing on graphs-part i: One-dimensional point processes. Neural Comput 2009;21(8):2152-202.

Dayan P, Abbot LF. Theoretical Neuroscience, Computational and Mathematical Modeling of Neural Systems. The MIT press, Cambridge, Massachusetts; 2001. 
Di Lorenzo PM, Chen JY, Victor JD. Quality Time: Representation of a Multidimensional Sensory Domain through Temporal Coding. J Neurosci 2009;29(29):9227-38.

Di Lorenzo PM, Victor JD. Taste response variability and temporal coding in the nucleus of the solitary tract of the rat. J Neurophysiol 2003;90(3):1418-31.

Di Lorenzo PM, Victor JD. Neural coding mechanisms for flow rate in tasteresponsive cells in the nucleus of the solitary tract of the rat. J Neurophysiol 2007;97(2):1857-61.

Gardner D. Neurodatabase.org: networking the microelectrode. Nature Neurosci 2004;7(5):486-87.

Gentner TQ. Temporal scales of auditory objects underlying birdsong vocal recognition. J Acoust Soc Am 2008;124(2):1350-59.

Grewe J, Kretzberg J, Warzecha AK, Egelhaaf M. Impact of photon noise on the reliability of a motion-sensitive neuron in the fly's visual system. J Neurosci 2003;23(34):10776-83.

Hallock RM, Di Lorenzo PM. Temporal coding in the gustatory system. Neurosci Behav Rev 2006;30(8):1145-60.

Houghton C, Sen K. A new multineuron spike train metric. Neural Comput 2008;20:1495-511.

Huetz C, Del Negro C, Lebas N, Tarroux P, Edeline JM. Contribution of spike timing to the information transmitted by hvc neurons. Eur J Neurosci 2006;24:1091-108.

Huetz C, Philibert B, Edeline JM. A spike-timing code for discriminating conspecific vocalizations in the thalamocortical system of anesthetized and awake Guinea pigs. J Neurosci 2009;29(2):334-50.

Hunter JD, Milton JG, Thomas PJ, Cowan JD. Resonance effect for neural spike time reliability. J Neurophysiol 1998;80(3):1427-38.

Jolivet R, Kobayashi R, Rauch A, Naud R, Shinomoto S, Gerstner W. A benchmark test for a quantitative assessment of simple neuron models. J Neurosci Meth 2008;169(2):417-24.

Kreiman G, Krahe R, Metzner W, Koch C, Gabbiani F. Robustness and variability of neuronal coding by amplitude-sensitive afferents in the weakly electric fish eigenmannia. J Neurophysiol 2000;84(1):189-204. 
Kreuz T, Chicharro D, Andrzejak RG, Haas JS, Abarbanel HDI. Measuring multiple spike train synchrony. J Neurosci Meth 2009;183(2):287-99.

Kreuz T, Chicharro D, Greschner M, Andrzejak RG. Time-resolved and time-scale adaptive measures of spike train synchrony. J Neurosci Meth 2011;195:92-106.

Kreuz T, Haas JS, Morelli A, Abarbanel HDI, Politi A. Measuring spike train synchrony. J Neurosci Meth 2007;165:151-61.

Machens CK, Prinz P, Stemmler MB, Ronacher B, Herz AVM. Discrimination of behaviorally relevant signals by auditory receptor neurons. Neurocomputing 2001;38-40:263-68.

Machens CK, Schütze H, Franz A, Kolesnikova O, Stemmler MB, Ronacher B, Herz AVM. Single auditory neurons rapidly discriminate conspecific communication signals. Nature Neurosci 2003;6:341-42.

Macleod K, Backer A, Laurent G. Who reads temporal information contained across synchronized and oscillatory spike trains? Nature 1998;395:693-98.

Mainen ZF, Sejnowski TJ. Reliability of spike timing in neocortical neurons. Science 1995;268(5216):1503-06.

Mechler F, Victor JD, Purpura KP, Shapley R. Robust temporal coding of contrast by v1 neurons for transient but not for steady-state stimuli. J Neurosci 1998;18(16):6583-98.

Nagel KI, Doupe AJ. Organizing principles of spectro-temporal encoding in the avian primary auditory area field 1 . Neuron 2008;58:938-35.

Narayan R, Ergun A, Sen K. Delayed inhibition in cortical receptive fields and the discrimination of complex stimuli. J Neurophysiol 2005;94(4):2970-75.

Narayan R, Graña GD, Sen K. Distinct time-scales in cortical discrimination of natural sounds in songbirds. J Neurophysiol 2006;96(1):252-58.

Nelken I, Chechik G, Mrsic-flogel TD, King AJ, Schnupp JWH. Encoding stimulus information by spike numbers and mean response time in primary auditory cortex. J Comput Neurosci 2005;19:199-221.

Paiva ARC, Park I, Principe JC. A reproducing kernel hilbert space framework for spike train signal processing. Neural Comput 2009;21(2):424-49.

Paiva ARC, Park I, Principe JC. A comparison of binless spike train measures. Neural Comput Appl 2010;19(3):405-19. 
Paninski L. Estimation of entropy and mutual information. Neural Comput 2003;15:1191-253.

Panzeri S, Petersen RS, Schultz SR, Lebedev M, Diamond ME. The role of spike timing in the coding of stimulus location in rat somatosensory cortex. Neuron 2001;29(3):769-77.

Panzeri S, Senatore R, Montemurro MA, Petersen RS. Correcting for the sampling bias problem in spike train information measures. J Neurophysiol 2007;98(3):1064-72.

Quian Quiroga R, Panzeri S. Extracting information from neuronal populations: information theory and decoding approaches. Nature Rev Neurosci 2009;10(3):173-85.

Reich DS, Mechler F, Victor JD. Formal and attribute-specific information in primary visual cortex. J Neurophysiol 2001a;85(1):305-18.

Reich DS, Mechler F, Victor JD. Temporal coding of contrast in primary visual cortex: When, what, and why. J Neurophysiol 2001b;85(3):1039-50.

Rieke F, Warland D, de Ruyter van Steveninck RR, Bialek W. Spikes: Exploring the neural code. MIT Press, Cambridge, Massachusetts; 1997.

Rolls ET, Treves A, Tovee MJ. The representational capacity of the distributed encoding of information provided by populations of neurons in primate temporal visual cortex. Exp Brain Res 1997;114(1):149-62.

Roussin AT, Victor JD, Chen JY, Di Lorenzo PM. Variability in responses and temporal coding of tastants of similar quality in the nucleus of the solitary tract of the rat. J Neurophysiol 2008;99(2):644-55.

Samonds JM, Bonds AB. From another angle: Differences in cortical coding between fine and coarse discrimination of orientation. J Neurophysiol 2004;91(3):1193-202.

Schneider DM, Woolley SMN. Discrimination of communication vocalizations by single neurons and groups of neurons in the auditory midbrain. J Neurophysiol 2010;103(6):3248-65.

Schnupp JWH, Hall TM, Kokelaar RF, Ahmed B. Plasticity of temporal pattern codes for vocalization stimuli in primary auditory cortex. J Neurosci 2006;26(18):4785-95. 
Schrauwen B, van Campenhout J. Linking non-binned spike train kernels to several existing spike train metrics. Neurocomputing 2007;70:1247-53.

Schreiber S, Fellous JM, Whitmer D, Tiesinga P, Sejnowski TJ. A new correlationbased measure of spike timing reliability. Neurocomputing 2003;52-54:925-31.

Shannon CE. A mathematical theory of communication. Bell Sys Tech J 1948;27:379-423, 623-56.

Strong SP, Koberle R, de Ruyter van Steveninck RR, Bialek W. Entropy and information in neural spike trains. Phys Rev Lett 1998;80:197-200.

Theunissen F, Miller JP. Temporal encoding in nervous systems: a rigorous definition. J Comput Neurosci 1995;2:149-62.

Theunissen FE, Sen K, Doupe AJ. Spectral-temporal receptive fields of nonlinear auditory neurons obtained using natural sounds. J Neurosci 2000;20:2315-31.

Thomson EE, Kristan WB. Quantifying stimulus discriminability: A comparison of information theory and ideal observer analysis. Neural Comput 2005;17(4):741-78.

Treves A, Panzeri S. The upward bias in measures of information derived from limited data samples. Neural Comput 1995;7(2):399-407.

van Rossum MCW. A novel spike distance. Neural Comput 2001;13:751-63.

Victor JD. Spike train metrics. Curr Opin Neurobiol 2005;15(5):585-92.

Victor JD. Approaches to information-theoretic analysis of neural activity. Biological Theory 2006;1(3):302-16.

Victor JD, Purpura KP. Nature and precision of temporal coding in visual cortex: A metric-space analysis. J Neurophysiol 1996;76:1310-26.

Victor JD, Purpura KP. Metric-space analysis of spike trains: theory, algorithms and application. Network: Comput Neural Syst 1997;8:127-64.

Victor JD, Purpura KP. Spatial phase and the temporal structure of the response to gratings in v1. J Neurophysiol 1998;80(2):554-71.

Wang L, Narayan R, Graña G, Shamir M, Sen K. Cortical discrimination of complex natural stimuli: Can single neurons match behavior? J Neurosci 2007;27(3):582-89.

Wohlgemuth S, Ronacher B. Auditory discrimination of amplitude modulations based on metric distances of spike trains. J Neurophysiol 2007;97(4):3082-92. 